\title{
Compactifications of deformed conifolds, branes and the geometry of qubits
}

\author{
M. Cvetič, ${ }^{a, f}$ G.W. Gibbons ${ }^{a, b, c, d}$ and C.N. Pope ${ }^{b, e}$ \\ ${ }^{a}$ Department of Physics and Astronomy, \\ University of Pennsylvania, Philadelphia, PA 19104, U.S.A. \\ ${ }^{b}$ DAMTP, Centre for Mathematical Sciences, \\ Cambridge University, Wilberforce Road, Cambridge CB3 OWA, U.K. \\ ${ }^{c}$ Laboratoire de Mathématiques et Physique Théorique CNRS-UMR 7350, \\ Fédération Denis Poisson, Université François-Rabelais Tours, \\ Parc de Grandmont, 37200 Tours, France \\ ${ }^{d}$ LE STUDIUM, Loire Valley Institute for Advanced Studies, \\ Tours and Orleans, France \\ ${ }^{e}$ George P. \& Cynthia W. Mitchell Institute for Fundamental Physics and Astronomy, \\ Texas A 6 M University, College Station, TX 7r843-4242, U.S.A. \\ ${ }^{f}$ Center for Applied Mathematics and Theoretical Physics, \\ University of Maribor, SI2000 Maribor, Slovenia \\ E-mail: cvetic@physics.upenn.edu, gwg1@cam.ac.uk, \\ pope@vmmail.physics.tamu.edu
}

ABSTRACT: We present three families of exact, cohomogeneity-one Einstein metrics in $(2 n+2)$ dimensions, which are generalizations of the Stenzel construction of Ricci-flat metrics to those with a positive cosmological constant. The first family of solutions are Fubini-Study metrics on the complex projective spaces $\mathbb{C P}^{n+1}$, written in a Stenzel form, whose principal orbits are the Stiefel manifolds $V_{2}\left(\mathbb{R}^{n+2}\right)=\mathrm{SO}(n+2) / \mathrm{SO}(n)$ divided by $\mathbb{Z}_{2}$. The second family are also Einstein-Kähler metrics, now on the Grassmannian manifolds $G_{2}\left(\mathbb{R}^{n+3}\right)=\mathrm{SO}(n+3) /((\mathrm{SO}(n+1) \times \mathrm{SO}(2))$, whose principal orbits are the Stiefel manifolds $V_{2}\left(\mathbb{R}^{n+2}\right)$ (with no $\mathbb{Z}_{2}$ factoring in this case). The third family are Einstein metrics on the product manifolds $S^{n+1} \times S^{n+1}$, and are Kähler only for $n=1$. Some of these metrics are believed to play a role in studies of consistent string theory compactifications and in the context of the AdS/CFT correspondence. We also elaborate on the geometric approach to quantum mechanics based on the Kähler geometry of Fubini-Study metrics on $\mathbb{C P}^{n+1}$, and we apply the formalism to study the quantum entanglement of qubits.

KeYwords: Conformal Field Models in String Theory, Models of Quantum Gravity, Differential and Algebraic Geometry

ARXiv EPRINT: 1507.07585 


\section{Contents}

1 Introduction 1

2 Quantum mechanics on $\mathbb{C P}^{n} \quad 4$

2.1 Darboux coordinates and shape space 5

$\begin{array}{lll}2.2 & \text { Entanglement and Segre embedding } & 7\end{array}$

2.3 Tripartite entanglement and Cayley hyperdeterminant 8

$\begin{array}{lll}2.4 & \text { Direct sums and nesting formulae } & 10\end{array}$

3 The Stenzel construction $\quad 10$

$4 \mathbb{C P}^{n+1}$ metrics in Stenzel form $\quad 13$

$\begin{array}{lll}4.1 & \text { Global structure of the } \mathbb{C P}^{n+1} \text { metrics } & 14\end{array}$

5 Other exact solutions of Stenzel form $\quad \mathbf{1 5}$

$\begin{array}{lll}5.1 & \text { Metrics on the Grassmannians } G_{2}\left(\mathbb{R}^{n+3}\right) & 15\end{array}$

$\begin{array}{lll}5.2 & \text { An } S^{n+1} \times S^{n+1} \text { solution of the second-order equations } & 17\end{array}$

$\begin{array}{lll}5.3 & \text { Non-compact manifolds with negative- } \Lambda \text { Einstein metrics } & 18\end{array}$

$\begin{array}{llr}6 & \text { Six dimensions } & \mathbf{1 8}\end{array}$

$\begin{array}{lll}\text { 6.1 Euler angles and fundamental domains } & 18\end{array}$

6.2 Comparison with numerical solution in [15] 21

7 Conclusions $\quad 22$

\section{Introduction}

The study of cohomogeneity-one Einstein metrics by employing the techniques used in homogeneous cosmology [1] was initiated in [2-5]. The Einstein equations lead to second-order differential equations which were shown to follow from a suitable Lagrangian. Imposing the condition that the metric have reduced holonomy was shown to lead to first-order differential equations that implied the second-order equations. In many cases these first-order equations admit simple explicit solutions. It was later shown that in many cases when this reduction is possible, the potential may be derived from a superpotential [6]. A particularly interesting class of examples consists of $(2 n+2)$-dimensional metrics with the isometry group $\mathrm{SO}(n+2)$, and these are the subject of the present paper. Specifically, the metrics we shall consider have cohomogeneity one, with level surfaces that are homogeneous squashed Stiefel manifolds $V_{2}\left(\mathbb{R}^{n+2}\right) \equiv O(n+2) / O(n) \equiv \mathrm{SO}(n+2) / \mathrm{SO}(n)$, consisting of the set of 
orthonormal dyads in $\mathbb{R}^{n+2}[7] .{ }^{1}$ In addition to the references cited above, some relevant previous work can be found in [8-13].

Perhaps the best-known example of a metric in the class we shall be considering is Stenzel's Ricci-flat 6-metric on the tangent bundle of the 3-sphere [10], which figures in string theory as the deformed conifold [14]. Recently, Kuperstein [15] has studied the behaviour of the conifold in the presence of a positive cosmological constant, and he found numerical evidence for a solution of a set of first-order equations that provides a complete non-singular cohomogeneity-one Einstein metric on a "compactification" of $T^{\star} S^{3}$. The 6 manifold is fibred by an open interval of five-dimensional principal orbits which degenerate at one end of the interval to an $S^{3}$ orbit, as in the case of the deformed conifold, and at the other end to an $S^{2} \times S^{2}$ orbit.

In this paper, we construct three families of simple exact solutions to the equations of motion for Stenzel-type Einstein metrics with a positive cosmological constant, and we study the global structures of the manifolds onto which these local metrics extend. Although the metrics are written in a cohomogeneity-one form, all three classes of metrics that we obtain are actually homogeneous. The first class of solutions we obtain, which satisfy the first-order equations and therefore are Einstein-Kähler, extend smoothly onto the manifolds of the complex projective spaces $\mathbb{C P}^{n+1}$. In fact, as we subsequently demonstrate, these are precisely the standard Fubini-Study metrics on $\mathbb{C P}^{n+1}$, but written in a rather unusual form. The principal orbits of these metrics are the Stiefel manifolds $V_{2}\left(\mathbb{R}^{n+2}\right)$, divided by $\mathbb{Z}_{2}$. The $\mathbb{C P}^{n+1}$ manifold is described in a form where there is an $S^{n+1}$ degenerate orbit or bolt at one end of the range of the cohomogeneity-one coordinate, and an $\mathrm{SO}(n+2) /(\mathrm{SO}(n) \times \mathrm{SO}(2)) / \mathbb{Z}_{2}$ bolt at the other end. The case $n=1$, giving $\mathbb{C P}^{2}$, corresponds to a solution of the first-order equations obtained by a geometrical construction presented in [9]. Here, we give a generalization of this construction to all values of $n$.

We find also a second family of exact solutions of the first-order equations. We demonstrate that these Einstein-Kähler metrics extend smoothly onto the Grassmannian manifolds $G_{2}\left(\mathbb{R}^{n+3}\right)=\mathrm{SO}(n+3) /\left((\mathrm{SO}(n+1) \times \mathrm{SO}(2))\right.$ of oriented 2-planes in $\mathbb{R}^{n+3}$. The level surfaces are again the Stiefel manifolds $V_{2}\left(\mathbb{R}^{n+2}\right) \equiv \mathrm{SO}(n+2) / \mathrm{SO}(n)$, which can be viewed as $\mathrm{U}(1)$ bundles over the Grassmannian manifolds $G_{2}\left(\mathbb{R}^{n+2}\right)=\mathrm{SO}(n+2) /(\mathrm{SO}(n) \times \mathrm{SO}(2))$. (In these metrics, unlike the $\mathbb{C P}^{n+1}$ metrics described above, the Stiefel manifolds of the principal orbits are not factored by $\mathbb{Z}_{2}$.) The metric we obtain on $G_{2}\left(\mathbb{R}^{n+3}\right)$ is homogeneous, described as a foliation of squashed Stiefel manifolds $V_{2}\left(\mathbb{R}^{n+2}\right)=\mathrm{SO}(n+2) / \mathrm{SO}(n)$. The metric has an $S^{n+1}$ bolt at at one end of the range of the cohomogeneity-one coordinate, just as in the Stenzel form of the $\mathbb{C P}^{n+1}$ metric, and an $\mathrm{SO}(n+2) /(\mathrm{SO}(n) \times \mathrm{SO}(2))$ bolt at the other end. The case $n=2$, corresponding to the Grassmannian $G_{2}\left(\mathbb{R}^{5}\right)$, is in fact the exact solution for an Einstein-Kähler metric that was found numerically by Kuperstein in [15].

\footnotetext{
${ }^{1}$ The reader is warned that there appears to be no standard notation for the Stiefel manifolds $V_{p}\left(\mathbb{R}^{n}\right)$ and their cousins the Grassmannian manifolds $G_{p}\left(\mathbb{R}^{n}\right)$. For us and in [7], the Stiefel manifold $V_{p}\left(\mathbb{R}^{p+q}\right)=O(p+q) / O(p)=\mathrm{SO}(p+q) / \mathrm{SO}(p)$ is the space of $p$-frames in $\mathbb{R}^{p+q}$. However, we differ from [7] on Grassmannian manifolds. For us $G_{p}\left(\mathbb{R}^{p+q}\right)=\mathrm{SO}(p+q) /(\mathrm{SO}(p) \times \mathrm{SO}(q))$ is the space of oriented $p$-planes in $\mathbb{R}^{p+q}$. In [7] $G_{p}\left(\mathbb{R}^{p+q}\right)=O(p+q) /(O(p) \times O(q))$ is the space of un-oriented $p$-planes in $\mathbb{R}^{p+q}$. The latter is a $\mathbb{Z}_{2}$ quotient of the former.
} 
The third family of metrics that we obtain arises as solutions of the second-order Einstein equations, but they do not, in general, satisfy the first-order equations. Thus they are Einstein but not Kähler. We provide a geometrical construction for those metrics, which demonstrates that they extend smoothly onto the product manifolds $S^{n+1} \times S^{n+1}$. In the case $n=1$, the geometrical construction coincides with one first given in [16] and described in detail in appendix $\mathrm{B}$ of [13]. The $n=1$ case is exceptional in that the metric, on $S^{2} \times S^{2}$, is Kähler as well as Einstein.

Some of the metrics discussed in this paper may play a role in studies of consistent M-theory or string theory compactifications, and in the context of the AdS/CFT correspondence. For example, a consistent compactification of Type IIA supergravity on $\mathbb{C P}^{3}$ results in an $N=6$ supersymmetric four-dimensional gauged supergravity theory. This was shown in [17], where it was obtained via a reduction of the $S^{7}$ compactification of $D=11$ supergravity on the Hopf fibres of the $S^{7}$ viewed as a $\mathrm{U}(1)$ bundle over $\mathbb{C P}^{3}$. The $\mathbb{C P}^{n+1}$ spaces also provide a natural base for constructions of elliptically fibered CalabiYau $(n+2)$-folds, relevant to studies of F-theory compactifications to $(8-2 n)$ dimensions (cf. [18] and references therein). In the context of the AdS/CFT correspondence, $\mathbb{C P}^{n+1}$ or $G_{2}\left(\mathbb{R}^{n+3}\right)$ backgrounds, as opposed to compact Calabi-Yau $(n+1)$-folds, have the possibility of avoiding the appearance of singular $\mathrm{D}-(p+2)$ brane fluxes in the presence of anti-D- $p$ branes (cf. [19] and references therein).

The relevance of the metrics discussed in this paper is not only restricted to problems in quantum gravity and in M-theory or string theory. The ideas presented in appendix B of [13] were taken from the quantum theory of triatomic molecules in the Born-Oppenheimer approximation. At a more fundamental level, $\mathbb{C P}^{n}$ is the space of physically distinct quantum states of a system with an $(n+1)$-dimensional Hilbert space, and forms the arena for the geometrical approach to quantum mechanics that exploits the Kähler geometry of its Fubini-Study metric [20-22]. The calculations on $\mathbb{C P}^{2}$ in [9] were aimed at evaluating the Aharonov-Anandan phase for a 3-state spin-1 system using the Kähler connection. More recently there have been interesting applications using ideas from toric geometry [23]. In this paper we shall further elaborate on applications of this formalism, including the study of the quantum entanglement of qubits.

The paper is organised as follows. In section 2 we give a brief outline of the geometric approach to quantum mechanics, and its further applications. This includes a discussion of the quantum entanglement of systems comprising two qubits and three qubits. In section 3 we summarise the Stenzel construction of the Ricci-flat metrics on the tangent bundle of $S^{n+1}$, which lends itself to the generalisation that allows us to construct Einstein-Kähler metrics with a positive cosmological constant. In section 4 we construct the explicit Einstein-Kähler metrics of the Stenzel type on $\mathbb{C P}^{n+1}$, and analyse their global structure. In section 5 we obtain the Einstein-Kähler metrics on the Grassmannian manifolds $G_{2}\left(\mathbb{R}^{n+3}\right)=\mathrm{SO}(n+3) /(\mathrm{SO}(n+1) \times \mathrm{SO}(2))$, as further exact solutions of the first-order equations for the metrics of Stenzel type. We also obtain exact solutions of the secondorder equations, for Einstein metrics of the Stenzel type that are not, in general, Kähler, on the product manifolds $S^{n+1} \times S^{n+1}$. Furthermore, by means of analytic continuations we obtain the metrics, with negative cosmological constant, on the non-compact forms of 
the $\mathbb{C} \mathbb{P}^{n+1}, G_{2}\left(\mathbb{R}^{n+3}\right)$ and $S^{n+1} \times S^{n+1}$ manifolds. In section 6 we discuss the case of six dimensions in detail, with an explicit coordinatisation of the left-invariant 1-forms on the five-dimensional principal orbits. We also provide a detailed comparison of our exact solutions with Kuperstein's numerical and asymptotic analysis. A summary and conclusions are given in section 7 .

\section{Quantum mechanics on $\mathbb{C P}^{n}$}

The goal of this section it to spell out the key steps in formulating a geometric approach to quantum mechanics, based on the Kähler geometry of the Fubini-Study metric on $\mathbb{C P}^{n}$. We begin by reminding the reader that in the standard formulation of quantum mechanics, Schrödinger's equation is just a special case of Hamilton's equations [24, 25]. Let $|a\rangle$, for $a=1,2 \ldots, n+1$, be an orthonormal basis for $\mathbb{C}^{n+1}$, and

$$
|\Psi\rangle=Z^{a}|a\rangle, \quad Z_{a}=\frac{1}{\sqrt{2}}\left(q^{a}+i p_{a}\right), \quad H\left(q^{a}, p_{a}, t\right)=\langle\Psi|\hat{H}| \Psi\rangle=\bar{Z}^{a} H_{a b} Z^{b},
$$

where $q^{a} \in \mathbb{R}^{n+1}, p_{a} \in \mathbb{R}^{n+1}$ and $H_{a b}=\langle a|\hat{H}| b\rangle=\bar{H}_{b a}$. Thus

$$
\frac{d Z^{a}}{d t}=\frac{1}{i} \frac{\partial H}{\partial \bar{Z}^{a}}
$$

or

$$
\frac{d q^{a}}{d t}=\frac{\partial H}{\partial p_{a}}, \quad \frac{d p_{a}}{d t}=-\frac{\partial H}{\partial q^{a}} .
$$

In effect, we are making use of the fact that $\mathbb{C}^{n+1}$, considered as a Hilbert space, is a flat Kähler manifold with Kähler potential $K=\bar{Z}^{a} Z^{a}$, metric

$$
\left.d s^{2}=|d| \Psi\right\rangle\left.\right|^{2}=\frac{\partial^{2} K}{\partial Z^{a} \partial \bar{Z}^{a}} d \bar{Z}^{a} d Z^{a}=d \bar{Z}^{a} d Z^{a}=\frac{1}{2}\left(d q^{a} d q^{a}+d p_{a} d p_{a}\right),
$$

symplectic form

$$
\omega=\frac{1}{i} \frac{\partial^{2} K}{\partial Z^{m} \partial \bar{Z}^{n}} d Z^{m} \wedge d \bar{Z}^{n}=\frac{1}{i} d \bar{Z}^{a} \wedge d \bar{Z}^{a}=d p_{a} \wedge d q^{a}
$$

and complex structure

$$
J \frac{d q^{a}}{d t}=\frac{d p_{a}}{d t}, \quad J \frac{d p_{a}}{d t}=-\frac{d q^{a}}{d t} .
$$

This formalism, however, has a built-in redundancy, since $|\Psi\rangle$ and $\lambda|\Psi\rangle$ with $\lambda$ a non-vanishing complex number are physically equivalent states. We can partially fix this freedom by normalising our states, requiring that

$$
\langle\Psi \mid \Psi\rangle=\bar{Z}^{a} Z^{a}=1
$$

This restricts the states to $S^{2 n+1} \subset \mathbb{R}^{2 n}$, but it still leaves the freedom to change the overall phase: $|\Psi\rangle \rightarrow e^{i \alpha}|\Psi\rangle$ with $\alpha \in \mathbb{R}$. To obtain the space of physically-distinct states, we must therefore take the quotient $S^{2 n+1} / \mathrm{U}(1)$. As a complex manifold this is just $\mathbb{C P}^{n}$, 
with the orbits of the $\mathrm{U}(1)$ action being the Hopf fibres. An atlas of complex coordinates is provided by the inhomogeneous coordinates $\zeta_{b}^{a}=Z^{a} / Z^{b}, a \neq b$.

In order to endow $\mathbb{C P}^{n}$ with a metric, we project the standard round metric on $S^{2 n+1}$ orthogonally to the fibres:

$$
\left.d s^{2}=|d| \Psi\right\rangle\left.\right|^{2}-|\langle\Psi|d| \Psi\rangle|^{2}=d \bar{Z}^{a} d Z^{a}-\left|\bar{Z}^{a} d Z^{a}\right|^{2} .
$$

Introducing the inhomogeneous coordinates $\zeta^{i}=Z^{i} / Z^{n+1}, i=1,2, \ldots, n$ we find that the Kähler form is given by

$$
K=\log \left(1+\bar{\zeta}^{i} \zeta^{i}\right) .
$$

If $n=1$ we get the Bloch sphere [26], with metric $\frac{1}{4}$ the unit round metric on $S^{2}$. This is the space of spin $\frac{1}{2}$ states, or of a single qubit. For a spin- $J$ state we get $\mathbb{C P}^{2 J}$. If $J=1$ one speaks of a q-trit and in general a q-dit with $d=(2 J+1)$. For $N$ qubits we have $n+1=d=2^{N}$, because in this case the Hilbert space is $\left(\mathbb{C}^{2}\right)^{\otimes N}$ and not $\left(S^{2}\right)^{N}$ as one might imagine for $N$ classical spin- $\frac{1}{2}$ particles.

The physical significance of the Fubini-Study metric is that the distance $s_{\mathrm{FB}}$ between two states $|\Psi\rangle$ and $\left|\Psi^{\prime}\right\rangle$ is given in terms of the transition probability $\left|\left\langle\Psi \mid \Psi^{\prime}\right\rangle\right|^{2}$ between the two states by

$$
\cos ^{2}\left(s_{\mathrm{FB}}\right)=\left|\left\langle\Psi \mid \Psi^{\prime}\right\rangle\right|^{2}
$$

Since in inhomogeneous coordinates

$$
|\Psi\rangle=\frac{1}{\sqrt{1+|\zeta|^{2}}}\left(\zeta^{i}|i\rangle+|n+1\rangle\right)
$$

we have

$$
\cos \left(s_{\mathrm{FB}}\right)=\frac{\left|1+\bar{\zeta}^{i} \zeta^{i}\right|}{\sqrt{\left(1+|\zeta|^{2}\right)\left(1+\left|\zeta^{\prime}\right|^{2}\right)}} .
$$

The instantaneous velocity of the evolution of a normalised state $|\Psi\rangle$ under the action of a Hamiltonian $\hat{H}$, which could be time-dependent is, using (2.8), given by

$$
\frac{d s_{\mathrm{FB}}}{d t}=\sqrt{\left\langle\Psi\left|\hat{H}^{2}\right| \Psi\right\rangle-(\langle\Psi|\hat{H}| \Psi\rangle)^{2}}=\Delta E,
$$

where $\Delta E$ is the instantaneous root mean square deviation of the energy in the state $|\Psi\rangle$. Note that (2.10) and (2.13) are discrepant by a factor of two from [27], whose metric is 4 times the Fubini-Study metric, that is $s_{A A}=2 s_{\mathrm{FB}}$.

\subsection{Darboux coordinates and shape space}

One may replace the inhomogeneous coordinates $\zeta^{i}$ by

$$
a^{i}=\frac{\zeta^{i}}{\sqrt{1+|\zeta|^{2}}}, \quad \Longleftrightarrow \quad \zeta^{i}=\frac{a^{i}}{\sqrt{1-|a|^{2}}},
$$

by which an open dense subset of $\mathbb{C P}^{n}$ is mapped into the interior of the unit ball in $\mathbb{C}^{n} \equiv \mathbb{R}^{2 n}$. Since if $K=\log \left(1+|\zeta|^{2}\right)$,

$$
\frac{1}{2} \frac{\partial^{2} K}{\partial \zeta^{i} \partial \bar{\zeta}^{j}} d \zeta^{i} \wedge d \bar{\zeta}^{j}=\frac{1}{2} d a^{i} \wedge d \bar{a}^{i}=i d p_{i} \wedge d q^{i}
$$


where $a^{k}=q^{k}+i p_{k}$. Thus $\left(q^{i}, p_{i}\right)$ are Darboux coordinates for $\mathbb{C P}^{n}$. If $n=1$ we recover what geographers call the coordinates associated to Lambert's Polar Azimuthal Equal Area Projection. By contrast, if $n=1$ and the inhomogeneous coordinate $\zeta^{1}$ is used, we have what astronomers and crystallographers know as The Equal Angle Stereographic Projection of Hipparchus.

In terms of the Lambert-Darboux coordinates we have

$$
|\Psi\rangle=a^{i}|i\rangle+\sqrt{1-|a|^{2}}|n+1\rangle,
$$

and hence $H=\langle\Psi|\hat{H}| \Psi\rangle$ is given by

$$
H=\bar{a}^{i} H_{i j} a^{j}+\left(1-|a|^{2}\right) H_{(n+1)(n+1)}+\sqrt{1-|a|^{2}}\left(\bar{a}^{i} H_{i(n+1)}+H_{(n+1) i} a^{i}\right),
$$

which is considerably simpler than its expression in inhomogeneous coordinates

$$
H=\frac{1}{\left(1+|\zeta|^{2}\right)}\left(\bar{\zeta}^{i} H_{i j} \zeta^{j}+\bar{\zeta}^{i} H_{i(n+1)}+H_{(n+1) i} \zeta^{i}+H_{(n+1)(n+1)}\right),
$$

In particular, if $H_{(n+1) i}=0$, the Hamiltonian is purely quadratic in the Lambert-Darboux coordinates. It is possible to express $\cos \delta_{F S}$ and the Fubini-Study metric in terms of Lambert-Darboux coordinates, but the expressions don't appear to be especially illuminating.

There is interesting application of the foregoing theory to the statistical theory of shape [28-30]. A shape is defined to be a set of $k$ labelled points $\mathbf{x}_{a}, a=1,2, \ldots n$ in $\mathbb{R}^{n}$ modulo the action of the similarity group $\operatorname{Sim}(n)$, i.e the group of translations, rotations and dilations. The space of such shapes is denoted by $\Sigma_{n}^{k}$ and hence has dimension $n k-n-\frac{1}{2} n(n-1)-1$. If we translate the $k$ points so that their centroid lies at the origin of $\mathbb{R}^{n}$, and we fix the scale by demanding that

$$
\sum_{1}^{k-1} \mathbf{x}_{i}^{2}=1
$$

we see that

$$
\Sigma_{n}^{k}=S^{n(k-1)-1} / \mathrm{SO}(n) .
$$

Moreover, the flat metric on $\mathbb{R}^{n(k-1)}$ descends to give a curved metric on $\Sigma_{n}^{k}$.

In the special case when $n=2$, we find that $\Sigma_{2}^{k}=S^{2 k-3} / \mathrm{SO}(2)=\mathbb{C P}^{k-2}$, with its Fubini-Study metric. Thus the space of triangles in the plane may be identified with the Bloch sphere $\mathbb{C P}^{1}$. Using complex notation, the $k-1$ coordinates $Z^{i}$, may be regarded as homogeneous coordinates for $\mathbb{C P}^{k-2}$. The inhomogeneous coordinates are $\zeta^{i}=Z^{i} / Z^{k-1}$, $i=1,2, \ldots, k-2$, and the Darboux coordinates are

$$
a^{i}=\frac{Z^{i}}{Z^{k-1}} \frac{1}{\sqrt{1+\sum_{j}^{k-2}\left|Z^{j}\right|^{2} /\left|Z^{k-1}\right|^{2}}}=e^{-i \theta_{k-1}} Z^{i}, \quad i=1,2, \ldots, k-2,
$$

where $\theta_{k-1}$ is the argument of $Z^{k-1}$. Thus if $e^{-i \theta_{k-1}} Z^{i}=x^{i}+i y^{i}$, the volume measure on the shape space $\Sigma_{2}^{k}$ is uniform in these Lambert-Darboux coordinates, i.e. it is

$$
\prod_{1}^{k-2} d x^{i} d y^{i}
$$


For a description of entanglement and other aspects of quantum mechanics in terms of shapes see [30].

\subsection{Entanglement and Segre embedding}

As noted above, the Hilbert space for two qubits is $\mathbb{C}^{2} \otimes \mathbb{C}^{2}=\mathbb{C}^{4}$, and the space of states is $\mathbb{C P}^{3}$, which as a real manifold is six dimensional. However, for two non-interacting completely independent spin-half systems, each of whose state spaces is the Bloch sphere $\mathbb{C P}^{1}=S^{2}$, one might expect a state space of the form $\mathbb{C P}^{1} \otimes \mathbb{C P}^{1}=S^{2} \times S^{2}$. This will be the case if we consider only separable or unentangled states in $\mathbb{C}^{4}=\mathbb{C}^{2} \otimes \mathbb{C}^{2}$, for which

$$
|\Psi\rangle=|\Psi\rangle_{1} \otimes|\Psi\rangle_{2}
$$

with

$$
|\Psi\rangle_{1}=a_{1}|\uparrow\rangle_{1}+b_{1}|\downarrow\rangle_{1}, \quad|\Psi\rangle_{2}=a_{2}|\uparrow\rangle_{2}+b_{2}|\downarrow\rangle_{2} .
$$

If $|\uparrow \uparrow\rangle=|\uparrow\rangle_{1} \otimes|\uparrow\rangle_{2}$, etc., then

$$
|\Psi\rangle=Z^{1}|\uparrow \uparrow\rangle+Z^{2}|\uparrow \downarrow\rangle+Z^{3}|\downarrow \uparrow\rangle+Z^{4}|\downarrow \downarrow\rangle,
$$

with

$$
\left(Z^{1}, Z^{2}, Z^{3}, Z^{4}\right)=\left(a_{1} a_{2}, a_{1} b_{2}, b_{1} a_{2}, b_{1} b_{2}\right),
$$

and so there is a non-linear constraint on the set of bi-partite states, namely

$$
Z^{1} Z^{4}=Z^{2} Z^{3}
$$

We conclude that the set of all separable states with respect to this factorization of the Hilbert space $\mathbb{C}^{4}$ is not a linear subspace of $\mathbb{C}^{4}$, but rather $(2.27)$ is a complex quadratic cone in $\mathbb{C}^{4}$. This projects down to a complex hypersurface in $\mathbb{C P}^{3}$, given, in terms of the inhomogeneous coordinates $\left(\zeta^{1}, \zeta^{2}, \zeta^{3}\right)=\left(Z^{1} / Z^{4}, Z^{2} / Z^{4}, Z^{3} / Z^{4}\right)$, by

$$
\zeta^{1}=\zeta^{2} \zeta^{3}
$$

The Kähler function for $\mathbb{C P}^{3}$ is

$$
K=\log \left(1+\left|\zeta^{1}\right|^{2}+\left|\zeta^{2}\right|^{2}+\left|\zeta^{3}\right|^{2}\right)
$$

and so this restricts to

$$
K=\log \left(1+\left|\zeta^{2} \zeta^{3}\right|^{2}+\left|\zeta^{2}\right|^{2}+\left|\zeta^{3}\right|^{2}\right)=\log \left(1+\left|\zeta^{2}\right|^{2}\right)+\log \left(1+\left|\zeta^{3}\right|^{2}\right) .
$$

Thus we get the product of Fubini-Study metrics on $\mathbb{C P}^{1} \times \mathbb{C P}^{1}$. This construction and its generalizations are known to mathematicians as Segre embeddings. In physical terms, a linear superposition of unentangled states is, in general, entangled. The span of all such states, that is, the union of complex lines on $\mathbb{C P}^{3}$ through all pairs of points on the Segre embedding of $\mathbb{C P}^{1} \times \mathbb{C P}^{1}$ into $\mathbb{C P}^{3}$, is all of $\mathbb{C P}^{3}$. 
The simplest notion of entanglement depends upon the factorization of the total Hilbert space into a tensor product of two Hilbert spaces. In our present case, since $2 \times 2=2+2$ the second Hilbert space is orthogonal,

$$
\mathcal{H}=\mathcal{H}_{\otimes} \mathcal{H}_{s}=\mathcal{H}_{1} \oplus_{\perp} \mathcal{H}_{2}
$$

Each factorization amounts to finding a two-dimensional linear subspace of $\mathbb{C}^{4}$. The space of such linear subspaces is is the complex Grassmannian $G_{2}\left(\mathbb{C}^{2}\right)=\mathrm{SU}(4) /\left(\mathrm{SU}(2)_{1} \times \mathrm{SU}(2)_{2}\right)$, where $\mathrm{SU}(2)_{1}$ acts on $\mathcal{H}_{1}$ and $\mathrm{SU}(2)_{2}$ acts on $\mathcal{H}_{2}$. In fact this is the only such simple case since the only integral solution of the equation $n_{1} n_{2}=n_{1}+n_{2}$ is $n_{1}=n_{2}=2$.

One physical situation where this decomposition arises is when $\mathrm{SU}(2)_{1}$ is isospin and $\mathrm{SU}(2)_{2}$ is ordinary spin. Then $|\uparrow\rangle \otimes|\Psi\rangle_{2}$ are states of the the proton with electric charge $|e|$ and $|\downarrow\rangle \otimes|\Psi\rangle_{2}$ are states of the neutron with zero electric charge [31]. Since electric charge is absolutely conserved, we have a super-selection rule [32]; no other superpositions are allowed. Thus the proton states correspond to a point at the north pole of $S_{1}^{2} \times S_{2}^{2}$ and the neutron states to a point at the south pole of $S_{1}^{2} \times S_{2}^{2}$.

\subsection{Tripartite entanglement and Cayley hyperdeterminant}

The significantly more complicated case of three qubits with the possibility of tripartite entanglement

$$
\mathbb{C}^{8}=\mathbb{C}^{2} \otimes \mathbb{C}^{2} \otimes \mathbb{C}^{2}
$$

which may be quantified by means the Cayley hyperdeterminant [33, 34], has arisen recently $[35,36]$ in the study of STU black holes $[37,38]$. If we adopt a binary digit notation, according to which $\uparrow$ corresponds to 0 and $\downarrow$ corresponds to 1 , we have

$$
\begin{aligned}
& \left(\zeta^{1}|0\rangle_{1}+|1\rangle_{1}\right) \otimes\left(\zeta^{2}|0\rangle_{2}+|1\rangle_{2}\right) \otimes\left(\zeta^{3}|0\rangle_{3}+|1\rangle_{3}\right) \\
= & \zeta^{000}|000\rangle+\zeta^{001}|001\rangle+\zeta^{100}|100\rangle \\
& +\zeta^{010}|010\rangle+\zeta^{110}|110\rangle+\zeta^{101}|101\rangle+\zeta^{011}|011\rangle+|111\rangle,
\end{aligned}
$$

where $\left(\zeta^{1}, \zeta^{2}, \zeta^{3}\right)$ are inhomogeneous coordinates for $\mathbb{C P}^{1} \times \mathbb{C P}^{1} \times \mathbb{C P}^{1}$ and $\left(\zeta^{000}, \ldots, \zeta^{011}\right)$ are inhomogeneous coordinates for $\mathbb{C P}^{7}$. In this case the Segre embedding is given (locally) by

$$
\begin{aligned}
\left(\zeta^{011}, \zeta^{101}, \zeta^{110}\right) & =\left(\zeta^{1}, \zeta^{2}, \zeta^{3}\right) \\
\left(\zeta^{001}, \zeta^{100}, \zeta^{010}\right) & =\left(\zeta^{1} \zeta^{2}, \zeta^{2} \zeta^{3}, \zeta^{3} \zeta^{1}\right) \\
\zeta^{000} & =\zeta^{1} \zeta^{2} \zeta^{3}
\end{aligned}
$$

or as a sub-variety of $\mathbb{C P}^{7}$ by the four equations in seven unknowns

$$
\begin{aligned}
\left(\zeta^{001}, \zeta^{010}, \zeta^{100}\right) & =\left(\zeta^{011} \zeta^{101}, \zeta^{011} \zeta^{110}, \zeta^{110} \zeta^{101}\right), \\
\zeta^{000} & =\zeta^{011} \zeta^{101} \zeta^{110} .
\end{aligned}
$$

In $[36]$ the general state in $\mathbb{C}^{8}$ is written as

$$
|\psi\rangle=\sum_{a} \psi_{a}|a\rangle
$$


where $a=0,1, \ldots, 7$ correspond to the binary digits used above. Thus

$$
\left(\psi_{0}, \psi_{1}, \psi_{2}, \psi_{3}, \psi_{4}, \psi_{5}, \psi_{6}, \psi_{7}\right)=\left(\zeta^{000}, \zeta^{001}, \zeta^{010}, \zeta^{011}, \zeta^{100}, \zeta^{101}, \zeta^{110}, 1\right)
$$

The Cayley hyperdeterminant is given by $[33,34]$

$$
D(\zeta)=-\frac{1}{2} b^{i j} b^{k \ell} \epsilon_{i k} \epsilon_{j \ell}, \quad \text { where } \quad b^{i j}=\zeta^{i k \ell} \zeta^{j m n} \epsilon_{k m} \epsilon_{\ell n}
$$

and $\epsilon_{i j}=-\epsilon_{j i}$ with $\epsilon_{01}=1$. In terms of the components $\psi_{a}$, this implies

$$
\begin{aligned}
D(|\psi\rangle)= & \left(\psi_{0} \psi_{7}-\psi_{1} \psi_{6}-\psi_{2} \psi_{5}-\psi_{3} \psi_{4}\right)^{2}-4\left(\psi_{1} \psi_{6} \psi_{2} \psi_{5}+\psi_{2} \psi_{5} \psi_{3} \psi_{4}+\psi_{3} \psi_{4} \psi_{1} \psi_{6}\right) \\
& +4 \psi_{1} \psi_{2} \psi_{4} \psi_{7}+4 \psi_{0} \psi_{3} \psi_{5} \psi_{6} .
\end{aligned}
$$

Substituting in (2.35), we see that the Cayley hyperdeterminant or three-tangle vanishes on the image of the Segre embedding, as expected. We can also see the embedding geometrically, in that the Kähler function for $\mathbb{C P}^{7}$,

$$
K_{7}=\log \left(1+\left|\zeta^{000}\right|^{2}+\left|\zeta^{001}\right|^{2}+\left|\zeta^{010}\right|^{2}+\left|\zeta^{011}\right|^{2}+\left|\zeta^{100}\right|^{2}+\left|\zeta^{101}\right|^{2}+\left|\zeta^{110}\right|^{2}\right),
$$

becomes the sum of Kähler functions for three $\mathbb{C P}^{1}$ factors after using the equations (2.35):

$$
K_{7} \longrightarrow \log \left(1+\left|\zeta^{1}\right|^{2}\right)+\log \left(1+\left|\zeta^{2}\right|^{2}\right)+\log \left(1+\left|\zeta^{3}\right|^{2}\right) .
$$

If the components $\psi_{a}$ are taken to be real, then the entropy of the BPS STU black holes [37, $38]$ and the Cayley hyperdeterminant are related by $[35,36]$ :

$$
S=\pi \sqrt{-D(|\psi\rangle)},
$$

provided that the four electric $\left\{q_{i}\right\}$ and four magnetic $\left\{p^{i}\right\}$ charges are identified as:

$$
\left(p^{0}, p^{1}, p^{2}, p^{3}, q_{0}, q_{1}, q_{2}, q_{3}\right)=\left(\psi_{0}, \psi_{1}, \psi_{2}, \psi_{4},-\psi_{7}, \psi_{6}, \psi_{5}, \psi_{3}\right) .
$$

$\mathbb{C}^{8}$ also admits a bi-partition as $\mathbb{C}^{2} \times \mathbb{C}^{4}$, and thus a Segre embedding of $\mathbb{C P}^{1} \times \mathbb{C P}^{3}$. This works out as follows. The analogue of (2.33) is

$$
\begin{aligned}
\left(\zeta^{0}|0\rangle_{1}+|1\rangle_{1}\right) & \otimes\left(\zeta^{1}|0\rangle_{2} \otimes|0\rangle_{3}+\zeta^{2}|1\rangle_{2} \otimes|0\rangle_{3}+\zeta^{3}|0\rangle_{2} \otimes|1\rangle_{3}+|1\rangle_{2} \otimes|1\rangle_{3}\right) \\
& =\sum_{a} \psi_{a}|a\rangle .
\end{aligned}
$$

The analogue of $(2.35)$ is

$$
\left(\psi_{0}, \psi_{1}, \psi_{2}, \psi_{3}, \psi_{4}, \psi_{5}, \psi_{6}, \psi_{7}\right)=\left(\zeta^{0} \zeta^{1}, \zeta^{0} \zeta^{3}, \zeta^{0} \zeta^{2}, \zeta^{0}, \zeta^{1}, \zeta^{3}, \zeta^{2}, 1\right),
$$

giving three equations in seven unknowns:

$$
\left(\psi_{0}, \psi_{1}, \psi_{2}\right)=\left(\psi_{3} \psi_{4}, \psi_{3} \psi_{5}, \psi_{3} \psi_{6}\right)
$$

or in other words

$$
\zeta^{000}=\zeta^{011} \zeta^{100}, \quad \zeta^{001}=\zeta^{011} \zeta^{101}, \quad \zeta^{010}=\zeta^{011} \zeta^{110} .
$$

Substitution of (2.45) in (2.39) shows that the Cayley hyperdeterminant of the three-tangle vanishes in this case as well. We also find that the Kähler function $(2.40)$ for $\mathbb{C P}^{7}$ becomes the sum of Kähler functions for a $\mathbb{C P}^{1}$ and a $\mathbb{C P}^{3}$ factor after imposing the conditions (2.46):

$$
K_{7} \longrightarrow \log \left(1+\left|\zeta^{0}\right|^{2}\right)+\log \left(1+\left|\zeta^{1}\right|^{2}+\left|\zeta^{2}\right|^{2}+\left|\zeta^{3}\right|^{2}\right) \text {. }
$$




\subsection{Direct sums and nesting formulae}

We have seen above that as well as partitions into tensor products, it is often convenient to decompose Hilbert spaces into direct sums. This gives rise to an iterative "nesting construction" for Fubini-Study metrics [39].

Consider the case

$$
\mathbb{C}^{p+q}=\mathbb{C}^{p} \oplus_{\perp} \mathbb{C}^{q}
$$

with $p \geq q$. Let

$$
Z=\left(\begin{array}{c}
\cos \alpha X \\
\sin \alpha Y
\end{array}\right)
$$

with

$$
X^{\dagger} X=Y^{\dagger} Y=1
$$

and hence $Z$ is a unit vector in $\mathbb{C}^{p+q}$ :

$$
Z^{\dagger} Z=1
$$

If we define $d \Sigma_{m}^{2}$, to be the Fubini-Study metric (2.8) on $\mathbb{C P}^{m}$, we have

$$
\begin{aligned}
d \Sigma_{p+q-1}^{2}= & d Z^{\dagger} d Z-\left|Z^{\dagger} d Z\right|^{2} \\
= & d \alpha^{2}+\cos ^{2} \alpha\left(d X^{\dagger} d X-\left|X^{\dagger} d X\right|^{2}\right)+\sin ^{2} \alpha\left(d Y^{\dagger} d Y-\left|Y^{\dagger} d Y\right|^{2}\right) \\
& +\cos ^{2} \alpha \sin ^{2} \alpha\left|X^{\dagger} d X+Y^{\dagger} d Y\right|^{2} \\
= & d \alpha^{2}+\cos ^{2} \alpha d \Sigma_{p-1}^{2}+\sin ^{2} \alpha d \Sigma_{q-1}^{2}+\sin ^{2} \alpha \cos ^{2} \alpha\left|X^{\dagger} d X+Y^{\dagger} d Y\right|^{2}
\end{aligned}
$$

where we have have used the fact that

$$
\Re X^{\dagger} d X=\Re Y^{\dagger} d Y=0 .
$$

Note that $-\mathrm{i} X^{\dagger} d X$ and $-\mathrm{i} Y^{\dagger} d Y$ are the Kähler connections on $\mathbb{C P}^{p-1}$ and $\mathbb{C P}^{q-1}$ respectively.

If $p=n, q=1, Y=e^{i \bar{\tau}}$ and $\alpha=\frac{1}{2} \pi-\xi$, we recover the iterative construction of [39], in which given the Fubini-Study metric on $\mathbb{C P}^{n}$, one obtains the Fubini-Study metric on $\mathbb{C P}^{n+1}$. Carrying out the iteration gives the metric as a nested sequence of metrics ending with the the round metric on $\mathbb{C P}^{1}$. In the first non-trivial case, one obtains $\mathbb{C P}^{2}$ in BianchiIX form [3]. It is clear that one may decompose the higher-dimensional metrics into further direct sums by using (2.54) applied to $d \Sigma_{q-1}^{2}$ or $d \Sigma_{q-1}^{2}$ or both.

\section{The Stenzel construction}

We begin by recalling the Stenzel construction of $(2 n+2)$-dimensional Ricci-flat metrics on the tangent bundle of $S^{n+1}$ [10]. It was described in detail, in a notation close to that which we shall be using here, in $[6] .{ }^{2}$ Let $L_{A B}$, which are antisymmetric in the

\footnotetext{
${ }^{2}$ The only change in notation is that we now take the index range for the $\mathrm{SO}(n)$ subgroup of $\mathrm{SO}(n+2)$ to be $1 \leq i \leq n$ rather than $3 \leq i \leq n+2$.
} 
fundamental $\mathrm{SO}(n+2)$ indices $A, B, \ldots$, be left-invariant 1-forms on the group manifold $\mathrm{SO}(n+2)$, obeying the exterior algebra

$$
d L_{A B}=L_{A C} \wedge L_{C B} .
$$

Splitting the indices $A=(i, n+1, n+2)$, the $L_{i j}$ are the left-invariant 1-forms of the $\mathrm{SO}(n)$ subgroup. We make the definitions of the 1-forms

$$
\sigma_{i} \equiv L_{i, n+1}, \quad \tilde{\sigma}_{i} \equiv L_{i, n+2}, \quad \nu \equiv L_{n+1, n+2},
$$

which lie in the coset $\mathrm{SO}(n+2) / \mathrm{SO}(n)$. They obey the algebra

$$
\begin{aligned}
d \sigma_{i} & =\nu \wedge \tilde{\sigma}_{i}+L_{i j} \wedge \sigma_{j}, \quad d \tilde{\sigma}_{i}=-\nu \wedge \sigma_{i}+L_{i j} \wedge \tilde{\sigma}_{j}, \quad d \nu=-\sigma_{i} \wedge \tilde{\sigma}_{i}, \\
d L_{i j} & =L_{i k} \wedge L_{k j}-\sigma_{i} \wedge \sigma_{j}-\tilde{\sigma}_{i} \wedge \tilde{\sigma}_{j} .
\end{aligned}
$$

We then consider the metric

$$
d s^{2}=d \xi^{2}+a^{2} \sigma_{i}^{2}+b^{2} \tilde{\sigma}_{i}^{2}+c^{2} \nu^{2},
$$

where $a, b$ and $c$ are functions of the radial coordinate $\xi$. We define also the vielbeins

$$
e^{0}=d \xi, \quad e^{i}=a \sigma_{i}, \quad e^{\tilde{i}}=b \tilde{\sigma}_{i}, \quad e^{\tilde{0}}=c \nu .
$$

The spin connection, curvature 2-forms and the Ricci tensor are given in [6]. It is also shown there that if one defines a new radial coordinate $\eta$ such that $a^{n} b^{n} c d \eta=d \xi$, then the Ricci-flat equations can be derived from the Lagrangian $L=T-V$ where

$$
\begin{aligned}
& T=\alpha^{\prime} \gamma^{\prime}+\beta^{\prime} \gamma^{\prime}+n \alpha^{\prime} \beta^{\prime}+\frac{1}{2}(n-1)\left(\alpha^{\prime 2}+\beta^{\prime 2}\right), \\
& V=\frac{1}{4}(a b)^{2 n-2}\left(a^{4}+b^{4}+c^{4}-2 a^{2} b^{2}-2 n\left(a^{2}+b^{2}\right) c^{2}\right),
\end{aligned}
$$

and $a=e^{\alpha}, b=e^{\beta}, c=e^{\gamma}$.

Writing the Lagrangian as $L=\frac{1}{2} g_{i j}\left(d \alpha^{i} / d \eta\right)\left(d \alpha^{j} / d \eta\right)-V$, where $\alpha^{i}=(\alpha, \beta, \gamma)$, the potential $V$ can be written in terms of a superpotential $W$, as [6]

$$
V=-\frac{1}{2} g^{i j} \frac{\partial W}{\partial \alpha^{i}} \frac{\partial W}{\partial \alpha^{j}}, \quad W=\frac{1}{2}(a b)^{n-1}\left(a^{2}+b^{2}+c^{2}\right) .
$$

(For a systematic discussion of when superpotentials can be introduced for the cohomogeneity-one Einstein equations, see [40,41].) This implies that the Ricci-flat conditions are satisfied if the first-order equations

$$
\frac{d \alpha^{i}}{d \eta}=g^{i j} \frac{\partial W}{\partial \alpha^{j}}
$$

are obeyed. This leads to the first-order equations [6]

$$
\dot{a}=\frac{1}{2 b c}\left(b^{2}+c^{2}-a^{2}\right), \quad \dot{b}=\frac{1}{2 a c}\left(a^{2}+c^{2}-b^{2}\right), \quad \dot{c}=\frac{n}{2 a b}\left(a^{2}+b^{2}-c^{2}\right),
$$

where $\dot{a}$ means $d a / d \xi$, etc. 
These first-order equations are in fact the conditions that follow from requiring that the metrics be Ricci-flat and Kähler, namely that $R_{a b}=0$ and that the Kähler form

$$
J=-e^{0} \wedge e^{\tilde{0}}+e^{i} \wedge e^{\tilde{i}}=-c d \xi \wedge \nu+a b \sigma_{i} \wedge \tilde{\sigma}_{i}
$$

be covariantly constant. In fact, they can be derived more simply by requiring

$$
d J=0, \quad d \Omega_{n+1}=0,
$$

where

$$
\Omega_{n+1} \equiv \epsilon^{0} \wedge \epsilon^{1} \wedge \cdots \wedge \epsilon^{n}
$$

is the holomorphic $(n+1)$-form and we have defined [6]

$$
\epsilon^{0} \equiv-e^{0}+\mathrm{i} e^{\tilde{0}}=-d \xi+\mathrm{i} c \nu, \quad \epsilon^{i} \equiv e^{i}+\mathrm{i} e^{\tilde{i}}=a \sigma_{i}+\mathrm{i} b \tilde{\sigma}_{i} .
$$

It is easy to incorporate a cosmological constant $\Lambda$, so that the equations of motion become $R_{a b}=\Lambda g_{a b}$. As was shown in [12], this Einstein condition is satisfied if the first-order equations (3.9) are modified to

$$
\dot{a}=\frac{1}{2 b c}\left(b^{2}+c^{2}-a^{2}\right), \quad \dot{b}=\frac{1}{2 a c}\left(a^{2}+c^{2}-b^{2}\right), \quad \dot{c}=\frac{n}{2 a b}\left(a^{2}+b^{2}-c^{2}\right)-\Lambda a b .
$$

These Einstein-Kähler first-order equations can also be derived by modifying the Ricci-flat Kähler conditions (3.11) to

$$
d J=0, \quad D \Omega_{n+1}=0,
$$

where $D$ is the $\mathrm{U}(1)$ gauge-covariant exterior derivative

$$
D \equiv d-\text { i } \Lambda A
$$

and $A$ is the Kähler 1-form potential, $J=d A$. From (3.10) and the equation $(a b)^{\prime}=c$ that follows from $d J=0$, it is easy to see that we can take

$$
A=-a b \nu
$$

The potential $V$ and superpotential $W$ appearing in (3.7) should be modified in the $\Lambda \neq 0$ case to

$$
\begin{aligned}
V & =\frac{1}{4}(a b)^{2 n-2}\left(a^{4}+b^{4}+c^{4}-2 a^{2} b^{2}-2 n\left(a^{2}+b^{2}\right) c^{2}+4 \Lambda a^{2} b^{2} c^{2}\right) \\
W & =\frac{1}{2}(a b)^{n-1}\left(a^{2}+b^{2}+c^{2}\right)-\frac{\Lambda}{n+1}(a b)^{n+1} .
\end{aligned}
$$

(The new superpotential for the special case $n=2$ was given in [15].) 


\section{$4 \mathbb{C P}^{n+1}$ metrics in Stenzel form}

We may now consider solutions of the first-order system of equations (3.14) for Einstein metrics of the Stenzel form. It is easy to see that for each value of $n$ there is a solution of (3.14) given by

$$
a=\sin \xi, \quad b=\cos \xi, \quad c=\cos 2 \xi
$$

with cosmological constant $\Lambda=2(n+2)$. (Of course, one can trivially apply scalings to obtain other values of the cosmological constant.)

As we shall now show, the metric (3.4) with $a, b$ and $c$ given by (4.1) is in fact the Fubini-Study metric on $\mathbb{C P}^{n+1}$, written in a non-standard way. To see this, we shall present the generalisation of a construction of $\mathbb{C P}^{2}$ given in [9], extended now to an arbitrary even dimension $D=2 n+2$.

Let $e_{n+1}$ and $e_{n+2}$ be an orthonormal pair of column vectors in $\mathbb{R}^{n+2}$, where

$$
e_{n+1}=(0,0, \ldots, 0,1,0)^{T}, \quad e_{n+2}=(0,0, \ldots, 0,0,1)^{T},
$$

and let $R$ be an arbitrary element of $\mathrm{SO}(n+2)$, which acts on $\mathbb{R}^{n+2}$ through matrix multiplication. We then define the complex $(n+2)$-vector

$$
Z=R\left(\sin \xi e_{n+1}+\mathrm{i} \cos \xi e_{n+2}\right),
$$

which clearly satisfies $Z^{\dagger} Z=1 .^{3}$ The standard construction of the Fubini-Study metric on $\mathbb{C P}^{n+1}$ is given, for $Z \in \mathbb{C}^{n+2}$ and satisfying $Z^{\dagger} Z=1$, by

$$
d s^{2}=d Z^{\dagger} d Z-\left|Z^{\dagger} d Z\right|^{2}
$$

Defining the 1-forms $L_{A B}$ on $\mathrm{SO}(n+2)$ by

$$
d R R^{-1}=\frac{1}{2} L_{A B} \widetilde{M}_{A B}
$$

where $\widetilde{M}_{A B}$ are the generators of the Lie algebra of $\mathrm{SO}(n+2)$, and introducing also the $\mathrm{SO}(n+2)$-conjugated generators

$$
M_{A B}=R^{T} \widetilde{M}_{A B} R
$$

we see from (4.3) that

$$
d Z=R\left[(L \cdot M)\left(\sin \xi e_{n+1}+\mathrm{i} \cos \xi e_{n+2}\right)+\left(\cos \xi e_{n+1}-\mathrm{i} \sin \xi e_{n+2}\right) d \xi\right],
$$

where we have defined $(L \cdot M)=\frac{1}{2} L_{A B} M_{A B}$. We may take the generators $M_{A B}$ to have components given simply by

$$
\left(M_{A B}\right)_{C D}=\delta_{A C} \delta_{B D}-\delta_{A D} \delta_{B C},
$$

\footnotetext{
${ }^{3}$ Since $Z$ and $-Z$ are the same point in $\mathbb{C P}^{n+1}$, this means that when $n$ is even (and hence $-R$ is in $\mathrm{SO}(n+2)$ if $R$ is in $\mathrm{SO}(n+2))$, the group that acts effectively on $\mathbb{C} \mathbb{P}^{n+1}$ is the projective special orthogonal group $P \mathrm{SO}(n+2)=\mathrm{SO}(n+2) / \mathbb{Z}_{2}$. By contrast, when $n$ is odd $\mathrm{SO}(n+2)$ is centreless, and so the entire $\mathrm{SO}(n+2)$ acts effectively on $\mathbb{C P}^{n+1}$.
} 
and so we can choose a basis where $e_{A}^{T}(L \cdot M) e_{B}=L_{A B}$. Note that the $L_{A B}$ are leftinvariant 1-forms of $\mathrm{SO}(n+2)$. It then follows that

$$
\begin{aligned}
Z^{\dagger} d Z & =\mathrm{i} \sin 2 \xi L_{n+1, n+2}, \\
d Z^{\dagger} d Z & =d \xi^{2}-\sin ^{2} \xi\left[(L \cdot M)^{2}\right]_{n+1, n+1}-\cos ^{2} \xi\left[(L \cdot M)^{2}\right]_{n+2, n+2},
\end{aligned}
$$

with

$$
\begin{aligned}
& {\left[(L \cdot M)^{2}\right]_{n+1, n+1}=L_{n+1, A} L_{A, n+1}=-\left(L_{n+1, n+2}\right)^{2}-\left(L_{i, n+1}\right)^{2},} \\
& {\left[(L \cdot M)^{2}\right]_{n+2, n+2}=L_{n+2, A} L_{A, n+2}=-\left(L_{n+1, n+2}\right)^{2}-\left(L_{i, n+2}\right)^{2} .}
\end{aligned}
$$

In view of the definitions (3.2), we therefore find that the Fubini-Study metric (4.4) on $\mathbb{C P}^{n+1}$ can be written as

$$
d s^{2}=d \xi^{2}+\sin ^{2} \xi \sigma_{i}^{2}+\cos ^{2} \xi \tilde{\sigma}_{i}^{2}+\cos ^{2} 2 \xi \nu^{2},
$$

which is precisely the metric we obtained above in (4.1).

The curvature 2-forms, which can be calculated from equations given in [6], turn out to be

$$
\begin{array}{ll}
\Theta_{0 i}=e^{0} \wedge e^{i}-e^{\tilde{0}} \wedge e^{\tilde{i}}, & \Theta_{0 \tilde{i}}=e^{0} \wedge e^{\tilde{i}}+e^{\tilde{0}} \wedge e^{i}, \\
\Theta_{0 \tilde{0}}=4 e^{0} \wedge e^{\tilde{0}}-2 e^{i} \wedge e^{\tilde{i}}, & \Theta_{i j}=e^{i} \wedge e^{j}+e^{\tilde{i}} \wedge e^{\tilde{j}}, \\
\Theta_{\tilde{i} \tilde{j}}=e^{\tilde{i}} \wedge e^{\tilde{j}}+e^{i} \wedge e^{j}, & \Theta_{i \tilde{j}}=e^{i} \wedge e^{\tilde{j}}-e^{\tilde{i}} \wedge e^{j}+2\left(e^{k} \wedge e^{\tilde{k}}-e^{0} \wedge e^{\tilde{0}}\right) \delta_{i j}, \\
\Theta_{\tilde{0} i}=e^{\tilde{0}} \wedge e^{i}+e^{0} \wedge e^{\tilde{i}}, & \Theta_{\tilde{0} \tilde{i}}=e^{\tilde{0}} \wedge e^{\tilde{i}}-e^{0} \wedge e^{i},
\end{array}
$$

where we are using the vielbein basis defined in (3.5). The $\mathbb{C} \mathbb{P}^{n+1}$ metrics are Einstein, with $R_{a b}=2(n+2) g_{a b}$. Note that as expected for the Fubini-Study metrics, the curvature has constant holomorphic sectional curvature, and can be written as

$$
\Theta_{A B}=e^{A} \wedge e^{B}+J_{A C} J_{B D} e^{C} \wedge e^{D}+2 J_{A B} J,
$$

where $J$ is the Kähler form, given in (3.10).

It will also be useful for future reference to note that the $\mathbb{C P}^{n+1}$ metric (4.11) can be rewritten in terms of a new radial coordinate $\tau=\log \tan \left(\xi+\frac{1}{4} \pi\right)$ as

$$
d s^{2}=\frac{1}{4} \operatorname{sech}^{2} \tau d \tau^{2}+\sinh ^{2} \frac{1}{2} \tau \operatorname{sech} \tau \sigma_{i}^{2}+\cosh ^{2} \frac{1}{2} \tau \operatorname{sech} \tau \tilde{\sigma}_{i}^{2}+\operatorname{sech}^{2} \tau \nu^{2} .
$$

The radial coordinate ranges from $\tau=0$ at the $S^{n+1}$ bolt to $\tau=\infty$ at the $\operatorname{SO}(n+$ $2) /(\mathrm{SO}(n) \times \mathrm{SO}(2)) / \mathbb{Z}_{2}$ bolt. [We note that in the appendix $\mathrm{A}$ of [42], an analogous, though less geometric construction of $\mathbb{C P}^{m+n+1}$ as a nesting of $\mathbb{C P}^{m} \times \mathbb{C P}^{n}$ surfaces was given.]

\subsection{Global structure of the $\mathbb{C P}^{n+1}$ metrics}

The radial coordinate $\xi$ lies in the interval $0 \leq \xi \leq \frac{1}{4} \pi$. As $\xi$ goes to zero, the metric (4.11) extends smoothly onto a space that has the local form $\mathbb{R}^{n+1} \times S^{n+1}$. As can be seen by 
comparing with the Ricci-flat Stenzel metrics as given in [6], the metrics take the same form in the vicinity of the origin. The principal orbits when $0<\xi<\frac{1}{4} \pi$ are the Stiefel manifold $\mathrm{SO}(n+2) / \mathrm{SO}(n)$ divided by $\mathbb{Z}_{2}$.

At the other end of the range of the $\xi$ coordinate, we see that as $\xi$ approaches $\frac{1}{4} \pi$, the metric (4.11) extends smoothly onto $\mathbb{R}^{2} \times G_{2}\left(\mathbb{R}^{n+2}\right) / \mathbb{Z}_{2}$. The reason for the $\mathbb{Z}_{2}$ quotient was discussed in footnote 3 . It is reflected in the fact that the integral $\oint \nu$ around the degenerate orbit at $\xi=\pi / 4$ must equal $\pi$, rather than $2 \pi$. This can be compared with the situation in metrics discussed in section 5.1 below, for which one has $\oint \nu=2 \pi$ at the analogous degenerate orbit.

In the language of nuts and bolts, the $\mathbb{C P}^{n+1}$ manifold is described here in a form where there is an $S^{n+1}$ degenerate orbit or bolt at $\xi=0$ and an $\mathrm{SO}(n+2) /(\mathrm{SO}(n) \times \mathrm{SO}(2)) / \mathbb{Z}_{2}$ bolt at $\xi=\frac{1}{4} \pi$.

Since $\oint \nu=\pi$ at the bolt, this implies that the level surfaces at fixed $\xi$ between the endpoints are the Stiefel manifold $\mathrm{SO}(n+2) / \mathrm{SO}(n)$ divided by $\mathbb{Z}_{2}$.

Although the local form of the $\mathbb{C} \mathbb{P}^{n+1}$ metrics near to the $S^{n+1}$ bolt at $\xi=0$ is similar to that of the Stenzel metrics on $T^{*} S^{n+1}$ near their $S^{n+1}$ bolt, the $\mathbb{Z}_{2}$ factoring of the $\mathrm{SO}(n+2) / \mathrm{SO}(n)$ principal orbits in the $\mathbb{C P}^{n+1}$ metrics that we discussed above means that one cannot, strictly speaking, view the $\mathbb{C P}^{n+1}$ metrics as "compactifications" of the Stenzel metrics. Rather, $\mathbb{C P}^{n+1}$ can be viewed as a "compactification" of the $\mathbb{Z}_{2}$ quotient of the Stenzel manifold. As can be seen from the construction of the Stenzel metrics given in section 2.1 of [6], where the Stenzel manifold is described by the complex quadric $z^{a} z^{a}=a^{2}$ in $\mathbb{C}^{n+2}$, one can divide by $\mathbb{Z}_{2}$, with the action $\mathbb{Z}_{2}: z^{a} \rightarrow-z^{a}$, and since this acts freely the quotient is still a smooth manifold. As a further cautionary remark, it should be noted that the Ricci-flat Stenzel metric on $T^{*} S^{n+1}$ does not arise as a limit of the $\mathbb{C P}^{n+1}$ metric (4.11) in which the cosmological constant is sent to zero.

\section{Other exact solutions of Stenzel form}

There are two other simple examples of Einstein metrics, with a positive cosmological constant $\Lambda$, that take the Stenzel form (3.4), on the manifolds $G_{2}\left(R^{n+3}\right)$ and $S^{n+1} \times S^{n+1}$. We present these in sections 5.1 and 5.2. In section 5.3, by making appropriate analytic continuations, we obtain Einstein metrics with negative cosmological constant on noncompact forms of $\mathbb{C P}^{n+1}, G_{2}\left(\mathbb{R}^{n+3}\right)$ and $S^{n+1} \times S^{n+1}$.

\subsection{Metrics on the Grassmannians $G_{2}\left(\mathbb{R}^{n+3}\right)$}

It is easy to see that the functions $a=\sin \xi, b=1, c=\cos \xi$ give a solution of the first-order equations (3.14), with $\Lambda=n+1$. This gives another Einstein-Kähler metric,

$$
d s^{2}=d \xi^{2}+\sin ^{2} \xi \sigma_{i}^{2}+\tilde{\sigma}_{i}^{2}+\cos ^{2} \xi \nu^{2} .
$$

The coordinate $\xi$ ranges from 0 to $\frac{1}{2} \pi$. Near $\xi=0$ the metric again looks locally like the Stenzel metric near its origin, and there is an $S^{n+1}$ bolt at $\xi=0$. The metric extends smoothly onto $\xi=\frac{1}{2} \pi$, provided that the integral $\oint \nu$ around $\xi=\pi / 2$ is equal to $2 \pi$. Thus in contrast to the $\mathbb{C P}^{n+1}$ metrics discussed in the previous section, 
where we found that regularity at the degenerate orbit required $\oint \nu=\pi$ and hence implied the non-degenerate level surfaces were $\mathrm{SO}(n+2) / \mathrm{SO}(n) / \mathbb{Z}_{2}$, in the present case the level surfaces are $\mathrm{SO}(n+2) / \mathrm{SO}(n)$. The bolt at $\xi=\frac{1}{2} \pi$ is the Grassmann manifold $G_{2}\left(\mathbb{R}^{n+2}\right)=\mathrm{SO}(n+2) /(\mathrm{SO}(n) \times \mathrm{SO}(2))$.

For future reference, we note that here if we introduce a new radial coordinate defined by $\tau=2 \log \tan \left(\frac{1}{2} \xi+\frac{1}{4} \pi\right)$, the metric (5.1) becomes

$$
d s^{2}=\frac{1}{4} \operatorname{sech}^{2} \frac{1}{2} \tau d \tau^{2}+\tanh ^{2} \frac{1}{2} \tau \sigma_{i}^{2}+\tilde{\sigma}_{i}^{2}+\operatorname{sech}^{2} \frac{1}{2} \tau \nu^{2} .
$$

The radial coordinate ranges from the $S^{n+1}$ bolt at $\tau=0$ to the $G_{2}\left(\mathbb{R}^{n+2}\right)$ bolt at $\tau=\infty$.

The curvature 2-forms, which can again be calculated from equations given in [6], turn out to be

$$
\begin{array}{rlrl}
\Theta_{0 i} & =e^{0} \wedge e^{i}+e^{\tilde{0}} \wedge e^{\tilde{i}}, & & \Theta_{0 \tilde{i}}=0, \\
\Theta_{0 \tilde{0}} & =e^{0} \wedge e^{\tilde{0}}+e^{i} \wedge e^{\tilde{i}}, & & \Theta_{i j}=e^{i} \wedge e^{j}+e^{\tilde{i}} \wedge e^{\tilde{j}}, \\
\Theta_{\tilde{i} \tilde{j}} & =e^{\tilde{i}} \wedge e^{\tilde{j}}+e^{i} \wedge e^{j}, & & \Theta_{i \tilde{j}}=\left(e^{k} \wedge e^{\tilde{k}}+e^{0} \wedge e^{\tilde{0}}\right) \delta_{i j}, \\
\Theta_{\tilde{0} i}=0, & & \Theta_{\tilde{0} \tilde{i}}=e^{\tilde{0}} \wedge e^{\tilde{i}}+e^{0} \wedge e^{i} .
\end{array}
$$

(We have chosen the vielbein basis $e^{0}=d \xi, e^{i}=\sin \xi \sigma_{i}, e^{\tilde{i}}=\tilde{\sigma}_{i}$ and $e^{\tilde{0}}=-\cos \xi \nu$ here.) Note that if we now define the indices

$$
I=(0, i), \quad \tilde{I}=(\tilde{0}, \tilde{i}), \quad 0 \leq I \leq n,
$$

where $\tilde{I}=I+n+1$, then the curvature 2 -forms in (5.3) can be written in the more compact form

$$
\Theta_{I J}=e^{I} \wedge e^{J}+e^{\tilde{I}} \wedge e^{\tilde{J}}, \quad \Theta_{\tilde{I} \tilde{J}}=e^{\tilde{I}} \wedge e^{\tilde{J}}+e^{I} \wedge e^{J}, \quad \Theta_{I \tilde{J}}=e^{K} \wedge e^{\tilde{K}} \delta_{I J} .
$$

From this it can be seen that the metrics (5.1) are Einstein, with $R_{a b}=(n+1) g_{a b}$.

The metrics (5.1) are in fact metrics on the Grassmannian manifolds

$$
G_{2}\left(\mathbb{R}^{n+3}\right)=\frac{\mathrm{SO}(n+3)}{\mathrm{SO}(n+1) \times \mathrm{SO}(2)} .
$$

This can be seen by starting from the left-invariant 1 -forms $\hat{L}_{A B}$ of $\mathrm{SO}(n+3)$, with $0 \leq$ $A \leq n+2$, decomposing the indices as $A=(I, a)$, where $I=0, \ldots, n$ and $a=n+1, n+2$, and then defining the $2(n+1)$-bein

$$
e^{I}=\hat{L}_{I, n+1}, \quad e^{\tilde{I}}=\hat{L}_{I, n+2},
$$

for the metric $d s^{2}=e^{I} \otimes e^{I}+e^{\tilde{I}} \otimes e^{\tilde{I}}$, where $\tilde{I}=I+n+1$. The spin connection is then given by

$$
\omega_{I J}=-\hat{L}_{I J}, \quad \omega_{\tilde{I} \tilde{J}}=-\hat{L}_{i j}, \quad \omega_{I \tilde{J}}=-\delta_{I J} \hat{L}_{n+1, n+2},
$$

and hence the curvature 2-forms are

$$
\Theta_{I J}=e^{I} \wedge e^{J}+e^{\tilde{I}} \wedge e^{\tilde{J}}, \quad \Theta_{\tilde{I} \tilde{J}}=e^{\tilde{I}} \wedge e^{\tilde{J}}+e^{I} \wedge e^{J}, \quad \Theta_{I \tilde{J}}=e^{K} \wedge e^{\tilde{K}} \delta_{I J} .
$$


Thus the curvature for these metrics on the Grassmannian manifolds $G_{2}\left(\mathbb{R}^{n+3}\right)$ is in precise agreement with the curvature (5.5) that we found for the metrics (5.1). It is easily verified that the 2 -form

$$
J=e^{I} \wedge e^{\tilde{I}}
$$

is closed, and furthermore covariantly constant, and hence it is a Kähler form for $G_{2}\left(\mathbb{R}^{n+3}\right)$.

Since the metrics (5.1) are locally similar to the Ricci-flat Stenzel metrics near the $S^{n+1}$ bolt at $\xi=0$, and the principal orbits for $\xi>0$ are the Stiefel manifolds $\mathrm{SO}(n+2) / \mathrm{SO}(n)$, just as in the Ricci-flat Stenzel metrics, one may view the metrics (5.1) as a kind of "compactification" of the Stenzel metrics. However, as we remarked earlier in the context of the $\mathbb{C P}^{n+1}$ metrics, one should view this interpretation with some caution, since there is no $\Lambda \rightarrow 0$ limit of the the metrics (5.1) that gives the Ricci-flat Stenzel metrics.

\subsection{An $S^{n+1} \times S^{n+1}$ solution of the second-order equations}

We can also find a solution of the second-order Einstein equations that is not a solution of the first-order equations (3.14), and thus it is not Kähler (at least with respect to the almost complex structure defined by $J$ in (3.10)). This is given by

$$
a=\sin \xi, \quad b=\cos \xi, \quad c=1,
$$

and it is Einstein with $\Lambda=2 n$. This is in fact the standard product metric on $S^{n+1} \times S^{n+1}$. This can be seen by introducing two orthonormal vectors in $\mathbb{R}^{n+2}$, as in (4.2), and then defining the two real $(n+2)$-vectors

$$
X=R\left(\sin \xi e_{n+1}+\cos \xi e_{n+2}\right), \quad Y=R\left(\sin \xi e_{n+1}-\cos \xi e_{n+2}\right),
$$

where $R$ is again a general element of $\mathrm{SO}(n+2)$. Note that these satisfy $X^{T} X=1$ and $Y^{T} Y=1$. The suitably scaled metric on $S^{n+1} \times S^{n+1}$ can be written as

$$
d s^{2}=\frac{1}{2} d X^{T} d X+\frac{1}{2} d Y^{T} d Y .
$$

Following analogous steps to those we used in the $\mathbb{C P}^{n+1}$ case, we find that here the metric on $S^{n+1} \times S^{n+1}$ becomes

$$
d s^{2}=d \xi^{2}+\sin ^{2} \xi \sigma_{i}^{2}+\cos ^{2} \xi \tilde{\sigma}_{i}^{2}+\nu^{2},
$$

which is precisely the one given by (5.11).

The coordinate $\xi$ here ranges over $0 \leq \xi \leq \frac{1}{2} \pi$. The metric has Stenzel-like behaviour near each endpoint, and can be viewed as a flow from an $S^{n+1}$ bolt at one end to a "slumped" $S^{n+1}$ bolt at the other end.

The curvature 2-forms are given by

$$
\begin{aligned}
\Theta_{0 i} & =e^{0} \wedge e^{i}+e^{\tilde{0}} \wedge e^{\tilde{i}}, & \Theta_{0 \tilde{i}} & =e^{0} \wedge e^{\tilde{i}}+e^{\tilde{0}} \wedge e^{i}, \\
\Theta_{0 \tilde{0}} & =0, & \Theta_{i j} & =e^{i} \wedge e^{j}+e^{\tilde{i}} \wedge e^{\tilde{j}}, \\
\Theta_{\tilde{i} \tilde{j}} & =e^{\tilde{i}} \wedge e^{\tilde{j}}+e^{i} \wedge e^{j}, & \Theta_{i \tilde{j}} & =e^{i} \wedge e^{\tilde{j}}+e^{\tilde{i}} \wedge e^{j}, \\
\Theta_{\tilde{0} i} & =e^{\tilde{0}} \wedge e^{i}+e^{0} \wedge e^{\tilde{i}}, & \Theta_{\tilde{0} \tilde{i}} & =e^{\tilde{0}} \wedge e^{\tilde{i}}+e^{0} \wedge e^{i},
\end{aligned}
$$

from which it can be seen that the metrics are Einstein, with $R_{a b}=2 n g_{a b}$. 


\subsection{Non-compact manifolds with negative- $\Lambda$ Einstein metrics}

By performing straightforward analytic continuations we can obtain Einstein metrics with negative cosmological constant on non-compact forms of all three classes of manifolds that we have considered in this paper. The procedure is the same in all three cases, and comprises the following steps. First, we perform a Wick rotation of the cohomogeneity-one coordinate $\xi$, sending $\xi \rightarrow \mathrm{i} \xi$. Next, we perform a Wick rotation on the coordinates $x^{A}$ of the $\mathbb{R}^{n+2}$ Euclidean space, sending $x^{n+2} \rightarrow \mathrm{i} x^{n+2}$. This has the effect of sending

$$
\sigma_{i} \longrightarrow \sigma_{i}, \quad \tilde{\sigma}_{i} \longrightarrow \mathrm{i} \tilde{\sigma}_{i}, \quad \nu \longrightarrow \mathrm{i} \nu
$$

Finally, we reverse the sign of the metric. The metrics (4.11), (5.1) and (5.14) then become

$$
\begin{aligned}
\widetilde{\mathbb{C P}^{n+1}}: & d s^{2}=d \xi^{2}+\sinh ^{2} \xi \sigma_{i}^{2}+\cosh ^{2} \xi \tilde{\sigma}_{i}^{2}+\cosh ^{2} 2 \xi \nu^{2}, \\
G_{2}\left(R^{n+3}\right): & d s^{2}=d \xi^{2}+\sinh ^{2} \xi \sigma_{i}^{2}+\tilde{\sigma}_{i}^{2}+\cosh ^{2} \xi \nu^{2}, \\
H^{n+1} \times H^{n+1}: & d s^{2}=d \xi^{2}+\sinh ^{2} \xi \sigma_{i}^{2}+\cosh ^{2} \xi \tilde{\sigma}_{i}^{2}+\nu^{2},
\end{aligned}
$$

where $\widetilde{\mathbb{C P}^{n+1}}$ and $\widetilde{G_{2}\left(R^{n+3}\right)}$ denote the non-compact forms of $\mathbb{C P}^{n+1}$ and $G_{2}\left(\mathbb{R}^{n+3}\right)$, and $H^{n+1}$ denotes the hyperbolic space that is the non-compact form of $S^{n+1}$. The left-invariant 1 -forms $\sigma_{i}, \tilde{\sigma}_{i}$ and $\nu$, which now span the coset $\mathrm{SO}(n+1,1) /(\mathrm{SO}(n) \times \mathrm{SO}(1,1))$, satisfy the exterior algebra

$$
\begin{aligned}
d \sigma_{i} & =-\nu \wedge \tilde{\sigma}_{i}+L_{i j} \wedge \sigma_{j}, \quad d \tilde{\sigma}_{i}=-\nu \wedge \sigma_{i}+L_{i j} \wedge \tilde{\sigma}_{j}, \quad d \nu=-\sigma_{i} \wedge \tilde{\sigma}_{i}, \\
d L_{i j} & =L_{i k} \wedge L_{k j}-\sigma_{i} \wedge \sigma_{j}+\tilde{\sigma}_{i} \wedge \tilde{\sigma}_{j} .
\end{aligned}
$$

The cosmological constants for the three metrics in (5.17) are given by $\Lambda=-2(n+2)$, $\Lambda=-(n+1)$ and $\Lambda=-2 n$, respectively. In each case the coordinate $\xi$ ranges from $\xi=0$ at the $S^{n+1}$ bolt to $\xi=\infty$.

\section{Six dimensions}

The case of six dimensions, corresponding to $n=2$, is of particular interest for a variety of applications in string theory. In this case the numerator group in the coset $\mathrm{SO}(n+$ $2) /(\mathrm{SO}(n) \times \mathrm{SO}(2))$ of the level surfaces of the Stenzel construction is $\mathrm{SO}(4)$, which is (locally) the product $\mathrm{SU}(2) \times \mathrm{SU}(2)$. In this section we introduce Euler angles and discuss their coordinate ranges. We also make a comparison of our six-dimensional exact solutions with the numerical results obtained in [15].

\subsection{Euler angles and fundamental domains}

The left-invariant $\mathrm{SO}(4)$ 1-forms $L_{A B}$ are related to two sets of left-invariant $\mathrm{SU}(2)$ 1-forms $\Sigma_{i}$ and $\widetilde{\Sigma}_{i}$ according to

$$
\begin{array}{lll}
\Sigma_{1}=L_{23}+L_{14}, & \Sigma_{2}=L_{31}+L_{24}, & \Sigma_{3}=L_{12}+L_{34}, \\
\widetilde{\Sigma}_{1}=L_{23}-L_{14}, & \widetilde{\Sigma}_{2}=L_{31}-L_{24}, & \widetilde{\Sigma}_{3}=L_{12}-L_{34} .
\end{array}
$$


These therefore satisfy

$$
d \Sigma_{i}=-\frac{1}{2} \epsilon_{i j k} \Sigma_{j} \wedge \Sigma_{k}, \quad d \widetilde{\Sigma}_{i}=-\frac{1}{2} \epsilon_{i j k} \widetilde{\Sigma}_{j} \wedge \widetilde{\Sigma}_{k}
$$

In view of the definitions (3.2), we therefore have that

$$
\begin{aligned}
\sigma_{1} & =-\frac{1}{2}\left(\Sigma_{2}+\widetilde{\Sigma}_{2}\right), & \sigma_{2} & =\frac{1}{2}\left(\Sigma_{1}+\widetilde{\Sigma}_{1}\right), \\
\tilde{\sigma}_{1} & =\frac{1}{2}\left(\Sigma_{1}-\widetilde{\Sigma}_{1}\right), & \tilde{\sigma}_{2} & =\frac{1}{2}\left(\Sigma_{2}-\widetilde{\Sigma}_{2}\right), \\
\nu & =\frac{1}{2}\left(\Sigma_{3}-\widetilde{\Sigma}_{3}\right), & L_{12} & =\frac{1}{2}\left(\Sigma_{3}+\widetilde{\Sigma}_{3}\right) .
\end{aligned}
$$

The $\mathrm{SU}(2)$ left-invariant 1-forms $\Sigma_{i}$ and $\widetilde{\Sigma}_{i}$ may be parameterised in terms of Euler angles $(\theta, \phi, \psi)$ and $(\tilde{\theta}, \tilde{\phi}, \widetilde{\psi})$ in the standard way:

$\Sigma_{\mathrm{F}} \sin \psi \sin \theta d \phi+\cos \psi d \theta, \Sigma_{2}=\cos \psi \sin \theta d \phi-\sin \psi d \theta, \Sigma_{3}=d \psi+\cos \theta d \phi$,

$\widetilde{\Sigma}_{\mathrm{F}} \sin \widetilde{\psi} \sin \tilde{\theta} d \tilde{\phi}+\cos \widetilde{\psi} d \tilde{\theta}, \widetilde{\Sigma}_{2}=\cos \widetilde{\psi} \sin \tilde{\theta} d \tilde{\phi}-\sin \widetilde{\psi} d \tilde{\theta}, \widetilde{\Sigma}_{3}=d \tilde{\psi}+\cos \tilde{\theta} d \tilde{\phi}$.

There are four inequivalent connected Lie groups whose Lie algebra is $\mathfrak{s o}(4)$, namely

$$
\mathrm{SU}(2) \times \mathrm{SU}(2), \quad \mathrm{SO}(4), \quad \mathrm{SU}(2) \times \mathrm{SO}(3), \quad \mathrm{SO}(3) \times \mathrm{SO}(3) .
$$

These are distinguished by their fundamental domains in the $(\psi, \widetilde{\psi})$ plane. We have

$$
\begin{array}{rll}
\mathrm{SU}(2) \times \mathrm{SU}(2): & 0 \leq \psi<4 \pi, & 0 \leq \widetilde{\psi}<4 \pi, \\
\mathrm{SO}(4): & 0 \leq \psi<4 \pi, & 0 \leq \widetilde{\psi}<4 \pi, \quad \text { and } \quad(\psi, \widetilde{\psi}) \equiv(\psi+2 \pi, \widetilde{\psi}+2 \pi), \\
\mathrm{SU}(2) \times \mathrm{SO}(3): & 0 \leq \psi<4 \pi, & 0 \leq \widetilde{\psi}<2 \pi, \\
\mathrm{SO}(3) \times \mathrm{SO}(3): & 0 \leq \psi<2 \pi, & 0 \leq \widetilde{\psi}<2 \pi .
\end{array}
$$

These identifications can be expressed in terms of the following generators:

$$
\begin{array}{ll}
T: & (\psi, \widetilde{\psi}) \longrightarrow(\psi+4 \pi, \widetilde{\psi}), \\
\widetilde{T}: & (\psi, \widetilde{\psi}) \longrightarrow(\psi, \widetilde{\psi}+4 \pi), \\
S: & (\psi, \widetilde{\psi}) \longrightarrow(\psi+2 \pi, \widetilde{\psi}), \\
\widetilde{S}: & (\psi, \widetilde{\psi}) \longrightarrow(\psi, \widetilde{\psi}+2 \pi), \\
D: & (\psi, \widetilde{\psi}) \longrightarrow(\psi+2 \pi, \widetilde{\psi}+2 \pi) .
\end{array}
$$

Clearly, these all commute, and they obey

$$
S^{2}=T, \quad \widetilde{S}^{2}=\widetilde{T}, \quad D^{2}=T \widetilde{T} .
$$

Starting from $(\psi, \widetilde{\psi})$ defined in $\mathbb{R}^{2}$, the four groups are obtained by quotienting by the action of the generators listed below:

$$
\begin{array}{rll}
\mathrm{SU}(2) \times \mathrm{SU}(2): & T, & \widetilde{T}, \\
\mathrm{SO}(4): & T, & \widetilde{T}, \quad D, \\
\mathrm{SU}(2) \times \mathrm{SO}(3): & T, & \widetilde{S}, \\
\mathrm{SO}(3) \times \mathrm{SO}(3): & S, & \widetilde{S} .
\end{array}
$$


Defining the oblique coordinates

$$
\psi_{ \pm}=\psi \pm \widetilde{\psi}
$$

the fundamental domains given above for the four cases can be re-expressed in terms of $\psi_{+}$ and $\psi_{-}$. This can be done straightforwardly by plotting the domain in the $(\psi, \widetilde{\psi})$ plane, partitioning where necessary into triangular sub-domains, and acting with the appropriate translation generators listed above in order to achieve a connected fundamental domain in the $\left(\psi_{+}, \psi_{-}\right)$plane. This gives

$$
\begin{aligned}
& \mathrm{SU}(2) \times \mathrm{SU}(2): 0 \leq \psi_{+}<8 \pi, \quad 0 \leq \psi_{-}<4 \pi, \\
& \mathrm{SO}(4): 0 \leq \psi_{+}<4 \pi, \quad 0 \leq \psi_{-}<4 \pi, \\
& \mathrm{SU}(2) \times \mathrm{SO}(3): 0 \leq \psi_{+}<8 \pi, \quad 0 \leq \psi_{-}<2 \pi, \\
& \mathrm{SO}(3) \times \mathrm{SO}(3): 0 \leq \psi_{+}<4 \pi, \quad 0 \leq \psi_{-}<2 \pi .
\end{aligned}
$$

Consider first the $\mathbb{C P}^{3}$ metric, given by (4.11) with $n=2$. Near the upper endpoint of the coordinate $\xi$, at $\xi=\pi / 4$, we may define $\xi=\pi / 4-\alpha$, and the metric approaches

$$
d s^{2} \rightarrow d \alpha^{2}+\alpha^{2}\left(d \psi_{-}+\cos \theta d \phi-\cos \tilde{\theta} d \tilde{\phi}\right)^{2}+\frac{1}{2}\left(\sigma_{i}^{2}+\tilde{\sigma}_{i}^{2}\right)
$$

This extends smoothly onto $\alpha=0$ provided that $\psi_{-}$is assigned the period

$$
\Delta \psi_{-}=2 \pi .
$$

Comparing with the periodicity conditions in (6.11) for $\mathrm{SO}(4)$, we see that the $\mathrm{SO}(4)$ group manifold is factored by $\mathbb{Z}_{2}$. This is consistent with the fact that $Z$, defined by (4.3) is equivalent to $-Z$ in $\mathbb{C P}^{3}$ : since $-R$ is in $\mathrm{SO}(4)$ if $R$ is in $\mathrm{SO}(4)$, we should identify $R$ and $-R$ in the construction (4.3), and hence we should impose (6.13). This identification divides $\mathrm{SO}(4)$ by its $\mathbb{Z}_{2}$ centre, giving the projective special orthogonal group $\operatorname{PSO}(4)=$ $\mathrm{SO}(3) \times \mathrm{SO}(3)$. Thus the principal orbits are $V_{2}\left(\mathbb{R}^{4}\right) / \mathbb{Z}_{2}$, where $V_{2}\left(\mathbb{R}^{4}\right)$ is the Stiefel manifold $\mathrm{SO}(4) / \mathrm{SO}(2)$.

Turning now to the metric on the six-dimensional Grassmannian manifold $G_{2}\left(\mathbb{R}^{5}\right)=$ $\mathrm{SO}(5) /(\mathrm{SO}(3) \times \mathrm{SO}(2))$, given by (5.1) with $n=2$, we see that near the upper end of the range of the $\xi$ coordinate, at $\xi=\pi / 2$, the metric takes the form

$$
d s^{2} \rightarrow d \alpha^{2}+\frac{1}{4} \alpha^{2}\left(d \psi_{-}+\cos \theta d \phi-\cos \tilde{\theta} d \tilde{\phi}\right)^{2}+\sigma_{i}^{2}+\tilde{\sigma}_{i}^{2},
$$

where we have written $\xi=\pi / 2-\alpha$. The metric extends smoothly onto $\alpha=0$ provided that $\psi_{-}$has the period

$$
\Delta \psi_{-}=4 \pi,
$$

and so from (6.11) we see that in this case the group acting on the $\xi=$ constant level surfaces is precisely $\mathrm{SO}(4)$. The principal orbits are the Stiefel manifold $V_{2}\left(\mathbb{R}^{4}\right)=\mathrm{SO}(4) / \mathrm{SO}(2)$, which is often called $T^{1,1}$.

Finally, in the case of the $S^{3} \times S^{3}$ metric given by (5.14) with $n=2$, it is evident from the general construction described in section 5.2 that the group acting on the level surfaces 
should be precisely $\mathrm{SO}(n+2)$, and thus when $n=2$ we should have $\Delta \psi_{-}=4 \pi$. This can by confirmed by noting from (5.15) that the metrics (5.14) satisfy $R_{a b}=2 n g_{a b}$ and thus when $n=2$ it must be isomorphic to the product metric on two 3 -spheres of radius $1 / \sqrt{2}$. Calculating the volume using the metric (5.14) then confirms that indeed we must have $\Delta \psi_{-}=4 \pi$. The principal orbits are the Stiefel manifold $V_{2}\left(\mathbb{R}^{4}\right)=\mathrm{SO}(4) / \mathrm{SO}(2)$.

\subsection{Comparison with numerical solution in [15]}

A solution of the first-order equations (3.14) in six dimensions was obtained recently by Kuperstein [15]. The left-invariant 1-forms on the five-dimensional principal orbits were denoted by $\left(g_{1}, g_{2}, g_{3}, g_{4}, g_{5}\right)$ in [15], and one can show that these may be related to our 1 -forms by

$$
g_{1}=\frac{1}{\sqrt{2}} \sigma_{1}, \quad g_{2}=\frac{1}{\sqrt{2}} \sigma_{2}, \quad g_{3}=-\frac{1}{\sqrt{2}} \tilde{\sigma}_{2}, \quad g_{4}=\frac{1}{\sqrt{2}} \tilde{\sigma}_{1}, \quad g_{5}=2 \nu .
$$

Comparing the metric given in eqn (2.1) of [15] with our metric (3.4), we see that the metric functions $e^{w}, e^{y}$ and $e^{z}$ in [15] are related to our metric functions $a, b$ and $c$ by

$$
e^{w}=\frac{3}{2} a^{2} b^{2} c^{2}, \quad e^{y}=\frac{a}{b}, \quad e^{z}=2 a b .
$$

The radial variable used in [15] is the same as the $\tau$ variable that we introduced in the rewriting of the $\mathbb{C P}^{n+1}$ metrics (4.14) and the $G_{2}\left(\mathbb{R}^{n+3}\right)$ metrics (5.2). Note that both for our $\mathbb{C P}^{3}$ and our $G_{2}\left(\mathbb{R}^{5}\right)$ metrics, we have $e^{y}=\tanh \frac{1}{2} \tau$, as in [15].

It is now a simple matter to compare the asymptotic forms of the metric functions found in the numerical solution in [15] with those for the exact solutions we have obtained in this paper. In particular, we see that near $\tau=0$ the function $e^{z}$ takes the form

$$
\begin{aligned}
\mathbb{C P}^{3}: & e^{z}=\tanh \tau=\tau-\frac{1}{3} \tau^{3}+\cdots, \\
G_{2}\left(\mathbb{R}^{5}\right): & e^{z}=2 \tanh \frac{1}{2} \tau=\tau-\frac{1}{12} \tau^{2}+\cdots .
\end{aligned}
$$

Ar large $\tau$, we have

$$
\begin{aligned}
\mathbb{C P}^{3}: & e^{z}=\tanh \tau=1-2 e^{-2 \tau}+2 e^{-4 \tau}+\cdots, \\
\left.G_{2}^{(} \mathbb{R}^{5}\right): & e^{z}=2 \tanh \frac{1}{2} \tau=2\left(1-2 e^{-\tau}+2 e^{-2 \tau}+\cdots\right) .
\end{aligned}
$$

Comparing with the asymptotic forms given in eqns (3.4) and (3.5) of [15], we see that the metric that was found numerically there coincides with our exact solution for the EinsteinKähler metric on the Grassmannian manifold $G_{2}\left(\mathbb{R}^{5}\right)=\mathrm{SO}(5) /(\mathrm{SO}(3) \times \mathrm{SO}(2))$, with the scale size $R=\sqrt{2 / 3}$, and the expansion coefficients $C_{I R}=1$, and $C_{U V}=-2$. Of course, one can trivially rescale our metric to obtain any desired value for $R .{ }^{4}$

\footnotetext{
${ }^{4}$ The coefficient $C_{U V}$ associated with the large- $\tau$ expansion in [15] is said to be approximately +1.96 in that paper, but clearly, given the form of the asymptotic expansion $e^{z}=3 R^{2}\left(1+C_{U V} e^{-\tau}+\frac{1}{2} C_{U V}^{2} e^{-2 \tau}+\cdots\right)$ appearing there, $C_{U V}$ must be negative rather than positive, since $e^{z}$ approaches $3 R^{2}$ from below rather than above, as $\tau$ goes to infinity.
} 


\section{Conclusions}

In this paper we constructed three classes of exact Einstein metrics of cohomogeneity one in $(2 n+2)$ dimensions. These are generalisations of the Stenzel construction of Ricci-flat metrics, in which a positive cosmological constant is introduced. We also studied the global structure of the manifolds onto which these local metrics extend.

- The first class of metrics, which satisfy the first-order Stenzel equations with a positive cosmological constant, are therefore Einstein-Kähler. We demonstrated that these metrics are the standard Fubini-Study metrics on the complex projective spaces $\mathbb{C P}^{n+1}$, though presented in an unusual form. The study of the global structure revealed that the principal orbits of these metrics are the Stiefel manifolds $V_{2}\left(\mathbb{R}^{n+2}\right)=\mathrm{SO}(n+2) / \mathrm{SO}(n)$ of 2 -frames in $\mathbb{R}^{n+1}$, quotiented by $\mathbb{Z}_{2}$. As the cohomogeneity-one coordinate approaches $\xi=0$, there is an $S^{n+1}$-dimensional degenerate orbit or bolt, while at $\xi=\frac{1}{4} \pi$ there is an $\mathrm{SO}(n+2) /(\mathrm{SO}(n) \times \mathrm{SO}(2)) / \mathbb{Z}_{2}$ degenerate orbit. The special case $n=1$, giving $\mathbb{C P}^{2}$, corresponds to a solution obtained by a geometrical construction in [9].

- The second class of metrics are also exact solutions of the first-order Stenzel equations with a positive cosmological constant. These homogeneous Einstein-Kähler metrics extend smoothly onto the Grassmannian manifolds $G_{2}\left(\mathbb{R}^{n+3}\right)=\mathrm{SO}(n+3) /((\mathrm{SO}(n+$ $1) \times \mathrm{SO}(2))$ of oriented 2-planes in $\mathbb{R}^{n+3}$, whose principal orbits are again the Stiefel manifolds $V_{2}\left(\mathbb{R}^{n+2}\right)$ (not factored by $\mathbb{Z}_{2}$ in this case), viewed as $\mathrm{U}(1)$ bundles over the Grassmannian manifolds $G_{2}\left(\mathbb{R}^{n+2}\right)$. The metric has an $S^{n+1}$ bolt at $\xi=0$, and an $\mathrm{SO}(n+2) /(\mathrm{SO}(n) \times \mathrm{SO}(2))$ bolt at $\xi=\frac{1}{2} \pi$. The case $n=2$, giving $G_{2}\left(\mathbb{R}^{5}\right)=$ $\mathrm{SO}(5) /(\mathrm{SO}(3) \times \mathrm{SO}(2))$, is a solution that was found numerically in [15]. It represents a generalisation of the conifold metric (6-dimensional Stenzel metric) to include a positive cosmological constant.

- The third class of Einstein metrics does not, in general, satisfy the first-order equations. A geometrical construction for these metrics demonstrates that they extend smoothly onto the product manifolds $S^{n+1} \times S^{n+1}$. The $n=1$ case was first constructed in [16] and described in detail in appendix B of [13]. This is also the only case in this class where the Einstein metric is also Kähler.

By making appropriate analytic continuations, we also obtained Einstein metrics with negative cosmological constant on non-compact forms of $\mathbb{C} \mathbb{P}^{n+1}, G_{2}\left(\mathbb{R}^{n+3}\right)$ and $S^{n+1} \times S^{n+1}$.

The compact Einstein spaces presented in this paper should play an important role in further studies of consistent compactifications of M-theory and string theory, as well in the context of the AdS/CFT correspondence. In addition to the $\mathbb{C P}^{3}$ metric in the Stenzel form, the role of the other classes of metrics with $n=2$, as well as those with $n>2$, deserves further investigation.

Kähler, but not Ricci flat, metrics on the deformed conifold arise in the theory of moduli space of $\mathbb{C P}^{1}$ lumps $[43,44]$. The method developed in this paper should be applicable to those metrics as well. 
In this paper we also treated the geometrical approach to quantum mechanics, where $\mathbb{C P}^{n}$ is the space of physically-distinct quantum states of a system with an $(n+1)$ dimensional Hilbert space, thus employing the geometry of its Fubini-Study metric [2022]. The calculations involving $\mathbb{C P}^{2}$ in [9] were aimed at evaluating the Aharonov-Anandan phase for a 3-state spin-1 system. We have elaborated further on the formalism, and spelled out applications to the discussion of quantum entanglement for systems with two qubits and three qubits. A linear superposition of two unentangled states is in general entangled. The set of such bi-partite states is spanned by the set of physically-distinct unentangled product states, which form the complex sub-variety $\mathbb{C P}^{1} \times \mathbb{C P}^{1} \subset \mathbb{C P}^{3}$, given in section 2.2 as an explicit Segre embedding.

The notion of entanglement depends on the factorisation of the total Hilbert space. In the case of two qubits the Hilbert space could be split into a product of two orthogonal two-dimensional subspaces, forming a complex Grassmannian manifold $G_{2}\left(\mathbb{C}^{2}\right)=$ $\mathrm{SU}(4) /\left(\mathrm{SU}(2)_{1} \times \mathrm{SU}(2)_{2}\right)$. An example of that type is a two qubit system consisting of nucleons, with $\mathrm{SU}(2)_{1}$ and $\mathrm{SU}(2)_{2}$ playing the role of isospin and spin symmetry respectively.

Studies of quantum entanglement for more complex systems, such as a three qubit system, are of great current interest, and the proposed geometric approach could shed further light on these important questions. Within this context, we studied the tripartite quantum entanglement of qubits, showing the vanishing of the Cayley hyperdeterminant.

Another area where the geometry of $\mathbb{C P}^{n}$ comes to the aid of physics is in quantum control theory [45].

\section{Acknowledgments}

The work of M.C. is supported in part by the DOE (HEP) Award DE-SC0013528, the Fay R. and Eugene L. Langberg Endowed Chair (M.C.) and the Slovenian Research Agency (ARRS). The work of G.W.G. was supported in part by the award of a LE STUDIUM Professorship held at the L.M.P.T. of the University of Francois Rabelais. The work of C.N.P. is supported in part by DOE grant DE-FG02-13ER42020. M.C. thanks the Cambridge Centre for Theoretical Cosmology, and G.W.G. and C.N.P. thank the UPenn Center for Particle Cosmology, for hospitality during the course of this work.

Open Access. This article is distributed under the terms of the Creative Commons Attribution License (CC-BY 4.0), which permits any use, distribution and reproduction in any medium, provided the original author(s) and source are credited.

\section{References}

[1] M.P. Ryan and L.C. Shepley, Homogeneous relativistic cosmologies, Princeton University Press, U.S.A. (1975).

[2] V.A. Belinsky, G.W. Gibbons, D.N. Page and C.N. Pope, Asymptotically euclidean Bianchi IX metrics in quantum gravity, Phys. Lett. B 76 (1978) 433 [InSPIRE].

[3] G.W. Gibbons and C.N. Pope, $C P^{2}$ as a gravitational instanton, Commun. Math. Phys. 61 (1978) 239 [INSPIRE]. 
[4] G.W. Gibbons and C.N. Pope, The positive action conjecture and asymptotically euclidean metrics in quantum gravity, Commun. Math. Phys. 66 (1979) 267 [INSPIRE].

[5] G.W. Gibbons, D.N. Page and C.N. Pope, Einstein metrics on $S^{3} R^{3}$ and $R^{4}$ bundles, Commun. Math. Phys. 127 (1990) 529 [inSPIRE].

[6] M. Cvetič, G.W. Gibbons, H. Lü and C.N. Pope, Ricci flat metrics, harmonic forms and brane resolutions, Commun. Math. Phys. 232 (2003) 457 [hep-th/0012011] [INSPIRE].

[7] A. Schwarz, Topology for physicists, Grundlehren der mathematischen Wissenschaften volume 308, Springer, Berlin Germany (1994).

[8] H. Pedersen, Eguchi-Hanson metrics with cosmological constant, Class. Quantum Grav. 2 (1985) 579.

[9] C. Bouchiat and G.W. Gibbons, Non-integrable quantum phase in the evolution of a spin 1 system: a physical consequence of the non-trivial topology of the quantum state space, $J$. Phys. (France) 49 (1988) 187.

[10] M.B. Stenzel, Ricci-flat metrics on the complexification of a compact rank one symmetric space, Manuscr. Math. 80 (1993) 151.

[11] A.S. Dancer and I.A.B. Strachan, Kähler-Einstein metrics with $\mathrm{SU}(2)$ action, Math. Proc. Camb. Phil. Soc. 115 (1994) 51325.

[12] A.S. Dancer and I.A.B. Strachan, Einstein metrics on tangent bundles of spheres, Class. Quant. Grav. 19 (2002) 4663 [math/0202297] [INSPIRE].

[13] M. Cvetič, G.W. Gibbons, H. Lü and C.N. Pope, Bianchi IX selfdual Einstein metrics and singular $G_{2}$ manifolds, Class. Quant. Grav. 20 (2003) 4239 [hep-th/0206151] [INSPIRE].

[14] P. Candelas and X.C. de la Ossa, Comments on conifolds, Nucl. Phys. B 342 (1990) 246 [INSPIRE].

[15] S. Kuperstein, A deformed conifold with a cosmological constant, JHEP 08 (2015) 026 [arXiv: 1504.02079] [INSPIRE].

[16] C.N. Pope, Instantons in quantum gravity, Ph.D. Thesis, Cambridge University, Cambridge, U.K. (1979).

[17] B.E.W. Nilsson and C.N. Pope, Hopf fibration of eleven-dimensional supergravity, Class. Quant. Grav. 1 (1984) 499 [INSPIRE].

[18] M. Cvetič, A. Grassi, D. Klevers and H. Piragua, Chiral four-dimensional F-theory compactifications with $\mathrm{SU}(5)$ and multiple $\mathrm{U}(1)$-factors, JHEP 04 (2014) 010 [arXiv: 1306.3987] [INSPIRE].

[19] F.F. Gautason, B. Truijen and T. Van Riet, Smeared antibranes polarise in AdS, JHEP 07 (2015) 165 [arXiv:1502.00927] [INSPIRE].

[20] T.W.B. Kibble, Geometrization of quantum mechanics, Commun. Math. Phys. 65 (1979) 189 [INSPIRE].

[21] G.W. Gibbons, Typical states and density matrices, J. Geom. Phys. 8 (1992) 147 [InSPIRE].

[22] D.C. Brody and L.P. Hughston, Geometric quantum mechanics, J. Geom. Phys. 38 (2001) 19 [quant-ph/9906086] [INSPIRE].

[23] Y. Aadel, A. Belhaj, Z. Benslimane, M.B. Sedra and A. Segui, Qubits from Adinkra graph theory via colored toric geometry, arXiv:1506.02523 [INSPIRE].

[24] P.A.M. Dirac, The quantum theory of the emission and absorption of radiation, Proc. Roy. Soc. Lond. A 114 (1927) 243. 
[25] F. Strochi, Complex coordinates and quantum mechanics, Rev. Mod. Phys. 38 (1966) 36.

[26] F. Bloch, Nuclear induction, Phys. Rev. 70 (1946) 7.

[27] J. Anandan and Y. Aharonov, Geometry of quantum evolution, Phys. Rev. Lett. 65 (1990) 1697 [InSPIRE].

[28] D.G. Kendall, Shape manifolds, procrustean metrics and complex-projective space, Bull. London Math. Soc. 16 (1984) 81.

[29] D.G. Kendall, D. Barden, T.K. Carne and H. Le, Shape and shape theory, Wiley Series in Probability and Statistics, Wiley (1999).

[30] D. Brody, Shapes of quantum states, J. Phys. A 37 (2004) 251.

[31] W. Heisenberg, Über den Bau der Atomkerne, Z. Phys. 77 (1932) 1.

[32] G.C. Wick, A.S. Wightman and E.P. Wigner, The intrinsic parity of elementary particles, Phys. Rev. 88 (1982) 101.

[33] A. Cayley, On the theory of linear transformations, Cambridge Math. J. 4 (1845) 1; also in Collected Papers volume 1, Cambride University Press, Cambridge, U.K. (1889).

[34] L.M. Gelfand, M.M. Kapranov and A.V. Zelevinsky, Discriminants, resultants and multi-dimensional determinants, Birkhäuser (1994).

[35] M.J. Duff, String triality, black hole entropy and Cayley's hyperdeterminant, Phys. Rev. D 76 (2007) 025017 [hep-th/0601134] [INSPIRE].

[36] L. Borsten, M.J. Duff and P. Levay, The black-hole/qubit correspondence: an up-to-date review, Class. Quant. Grav. 29 (2012) 224008 [arXiv:1206.3166] [InSPIRE].

[37] M. Cvetič and D. Youm, Dyonic BPS saturated black holes of heterotic string on a six torus, Phys. Rev. D 53 (1996) 584 [hep-th/9507090] [InSPIRE].

[38] M. Cvetič and A.A. Tseytlin, Solitonic strings and BPS saturated dyonic black holes, Phys. Rev. D 53 (1996) 5619 [Erratum ibid. D 55 (1997) 3907] [hep-th/9512031] [INSPIRE].

[39] P. Hoxha, R.R. Martinez-Acosta and C.N. Pope, Kaluza-Klein consistency, Killing vectors and Kähler spaces, Class. Quant. Grav. 17 (2000) 4207 [hep-th/0005172] [INSPIRE].

[40] A. Dancer and M. Wang, Superpotentials and the cohomogeneity one Einstein equations, Commun. Math. Phys. 260 (2005) 75.

[41] A. Dancer and M. Wang, Classifying superpotentials: three summands case, J. Geom. Phys. 61 (2011) 675 [inSPIRE].

[42] M. Cvetič, H. Lü and C.N. Pope, Consistent warped space Kaluza-Klein reductions, half maximal gauged supergravities and $C P^{n}$ constructions, Nucl. Phys. B 597 (2001) 172 [hep-th/0007109] [INSPIRE].

[43] J.M. Speight, The Deformed conifold as a geometry on the space of unit charge CP ${ }^{1}$ lumps, Phys. Lett. B 511 (2001) 295 [hep-th/0105142] [INSPIRE].

[44] J.M. Speight, The $L^{* * 2}$ geometry of spaces of harmonic maps $S^{2} \rightarrow S^{2}$ and $R P^{2} \rightarrow R P^{2}$, J. Geom. Phys. 47 (2003) 343 [math/0102038] [InSPIRE].

[45] D.C. Brody, G.W. Gibbons and D.M. Meier, Time-optimal navigation through quantum wind, New J. Phys. 17 (2015) 033048 [arXiv:1410.6724] [INSPIRE]. 